\title{
Oxygen-sensing PHDs regulate bone homeostasis through the modulation of osteoprotegerin
}

\author{
Colleen Wu, ${ }^{1}$ Erinn B. Rankin, ${ }^{1,2}$ Laura Castellini, ${ }^{1}$ Javier Fernandez-Alcudia ${ }^{3}$ Edward L. LaGory, ${ }^{1}$ \\ Rebecca Andersen, ${ }^{1,8,9}$ Steven D. Rhodes, ${ }^{4,5}$ Tremika L.S. Wilson, ${ }^{6,10,11}$ Khalid S. Mohammad, ${ }^{4,6}$ \\ Alesha B. Castillo, ${ }^{7,12}$ Theresa A. Guise, ${ }^{6}$ Ernestina Schipani, ${ }^{6,10,11}$ and Amato J. Giaccia ${ }^{1}$ \\ ${ }^{1}$ Division of Cancer and Radiation Oncology, Department of Radiation Oncology, Stanford University, Stanford, California 94305, \\ $\mathrm{USA}_{;}{ }^{2}$ Department of Obstetrics and Gynecology, Stanford University, Stanford, California 94305, USA; ${ }^{3}$ Department of \\ Dermatology, Stanford University, Stanford, California 94305, USA; ${ }^{4}$ Department of Anatomy and Cell Biology, ${ }^{5}$ Department of \\ Medicine, Indiana School of Medicine, Indianapolis, Indiana 46202, USA; ${ }^{6}$ Division of Endocrinology, Department of Medicine, \\ Indiana School of Medicine, Indianapolis, Indiana 46202, USA; ${ }^{7}$ Division of Plastic and Reconstructive Surgery, Department of \\ Surgery, Stanford University, Stanford, California 94305, USA
}

The bone microenvironment is composed of niches that house cells across variable oxygen tensions. However, the contribution of oxygen gradients in regulating bone and blood homeostasis remains unknown. Here, we generated mice with either single or combined genetic inactivation of the critical oxygen-sensing prolyl hydroxylase (PHD) enzymes (PHD1-3) in osteoprogenitors. Hypoxia-inducible factor (HIF) activation associated with Phd2 and Phd3 inactivation drove bone accumulation by modulating osteoblastic/osteoclastic cross-talk through the direct regulation of osteoprotegerin (OPG). In contrast, combined inactivation of Phd1,Phd2, and Phd3 resulted in extreme HIF signaling, leading to polycythemia and excessive bone accumulation by overstimulating angiogenic-osteogenic coupling. We also demonstrate that genetic ablation of $P h d 2$ and $P h d 3$ was sufficient to protect ovariectomized mice against bone loss without disrupting hematopoietic homeostasis. Importantly, we identify OPG as a HIF target gene capable of directing osteoblast-mediated osteoclastogenesis to regulate bone homeostasis. Here, we show that coordinated activation of specific PHD isoforms fine-tunes the osteoblastic response to hypoxia, thereby directing two important aspects of bone physiology: cross-talk between osteoblasts and osteoclasts and angiogenic-osteogenic coupling.

[Keywords: HIF signaling; bone homeostasis; hypoxia; osteoprotegerin; oxygen sensing; prolyl hydroxylase]

Supplemental material is available for this article.

Received October 28, 2014; revised version accepted March 11, 2015.

Bone development and homeostasis are achieved through an intricate balance between osteoblastic bone formation and osteoclastic bone resorption (Zaidi 2007; Khosla et al. 2008). Mammalian bone develops through endochondral ossification, a process that requires the coordination of osteoblasts and blood vessel invasion into the cartilaginous template to trigger the formation of the primary ossification center, highlighting the importance of angiogenesis during bone formation (Kronenberg 2003; Provot and Schipani 2005; Maes 2013). Maintenance of bone homeostasis is achieved by coupling bone formation with osteoblastic

Present addresses: ${ }^{8}$ Department of Neurological Surgery, ${ }^{9}$ Department of Surgery, University of California at San Francisco, CA 94101, USA; ${ }^{10}$ Department of Orthopedic Surgery, ${ }^{11}$ Department of Medicine/Endocrinology, University of Michigan, Ann Arbor, MI 98109, USA; ${ }^{12}$ Department of Mechanical and Aerospace Engineering, New York University, Brooklyn, NY 11201, USA.

Corresponding author: giaccia@stanford.edu

Article published online ahead of print. Article and publication date are online at http://www.genesdev.org/cgi/doi/10.1101/gad.255000.114. bone resorption. In particular, the RANK/RANKL/osteoprotegerin (OPG) axis contributes to this process by facilitating osteoblast-mediated osteoclastogenesis (Boyle et al. 2003). Deregulation of bone homeostasis is a hallmark of pathophysiological bone disease and occurs due to imbalances in osteogenic-angiogenic coupling or between bone-forming osteoblasts and bone resorption by osteoclasts. Increased bone results in osteopetrosis, whereas osteoporosis is associated with bone loss (Teitelbaum and Ross 2003; Seeman and Delmas 2006). Elucidating the molecular mechanisms that control bone homeostasis within the bone microenvironment is needed in order to identify novel therapeutic strategies to

(C) $2015 \mathrm{Wu}$ et al. This article is distributed exclusively by Cold Spring Harbor Laboratory Press for the first six months after the full-issue publication date (see http://genesdev.cshlp.org/site/misc/terms.xhtml). After six months, it is available under a Creative Commons License (Attribution-NonCommercial 4.0 International), as described at http:// creativecommons.org/licenses/by-nc/4.0/. 
generate functional bone to treat chronic metabolic bone disorders where the options for treatment are still limited.

The bone microenvironment is a particularly hypoxic microenvironment. Most adult tissues are well vascularized, allowing for the promotion of oxygen transport with normal median interstitial oxygen tension $\left(\mathrm{pO}_{2}\right)$ values ranging from $3 \%$ to $9 \%$ (Vaupel et al. 1989). In contrast, $\mathrm{pO}_{2}$ levels in the bone range from $<1 \%$ in hypoxic regions up to $6 \%$ in sinusoidal cavities (Mohyeldin et al. 2010). Low oxygen tensions in the bone are likely a result of low oxygen levels in sinusoidal blood and high levels of oxygen consumption by hematopoietic cells. Blood flow in the bone travels from the central artery into arterioles, capillaries, and finally sinusoids, accompanied by a progressive drop in blood velocity and oxygenation. It has been estimated that the blood flow rate in sinusoids is approximately $1 / 10$ to $1 / 20$ of that found in arterioles. In addition to low blood flow and oxygen tension within sinusoids, mathematical models predict that a layer of three myeloid progenitors is sufficient to consume most oxygen provided by a nearby sinusoid (Chow et al. 2001; Winkler et al. 2010, Rankin et al. 2011). Immunohistochemical staining and microcomputer tomography $(\mu \mathrm{CT})$ imaging of bone demonstrate that blood vessels within the adult bone and bone marrow display large structural and spatial distributional heterogeneity (Kusumbe et al. 2014; Regan et al. 2014; Spencer et al. 2014). Hence, this tissue displays vascular heterogeneity resulting in regionally defined areas of low oxygen tension or hypoxia. Despite the fact that the bone resides in a hypoxic microenvironment, the contribution of this oxygen gradient and the role of the cellular oxygen sensors in regulating bone homeostasis remain poorly understood.

Prolyl hydroxylase (PHD) enzymes (PHD1, PHD2, and PHD3) are the primary cellular oxygen sensors (Semenza 2001). Most notably, PHD enzymes use oxygen as a molecular substrate to regulate the protein stability of the hypoxia-inducible transcription factors (HIF-1 $\alpha$ and HIF$2 \alpha$ ) in an oxygen-dependent manner (Schofield and Ratcliffe 2004). At oxygen tensions $>5 \%$, the PHD enzymes regulate HIF protein stability by catalyzing the hydroxylation of HIF transcription factors on conserved proline residues, targeting them for proteosomal degradation by the von Hippel-Lindau (pVHL) ubiquitin ligase complex. When oxygen tensions fall below $5 \%$, PHD enzyme activity is diminished, and HIF- $1 \alpha$ and HIF- $2 \alpha$ are stabilized and activate the expression of genes involved in diverse physiological conditions ranging from erythropoiesis to metabolism to angiogenesis (Semenza 2012). Thus, PHD enzymes provide a direct link between oxygen availability and the regulation of HIF transcriptional activity. While the PHD isoforms have nearly ubiquitous tissue expression, the tissue-specific role of each isoform in regulating HIF protein levels remains poorly defined.

Here, we sought to dissect the physiologic role of oxygen-sensing PHD1, PHD2, and PHD3 enzymes in the regulation of bone homeostasis. For this purpose, we used a genetic approach to specifically inactivate Phd1, Phd2, and $P h d 3$ in cells of the osteoblastic lineage using CreloxP-mediated recombination. In addition to coordinating osteoblastic-angiogenic coupling, we identified a previously unknown role for the PHD/HIF signaling pathway in the osteoblastic regulation of osteoclastogenesis through the direct regulation of OPG. Importantly, inactivation of selective PHD isoforms within the cells of the osteoblastic lineage inhibited bone loss in ovariectomized mice. Thus, molecular oxygen, through the PHD/HIF signaling pathway, plays a central role in bone homeostasis by controlling both angiogenesis and osteoclastogenesis, and therapeutic targeting of PHD enzymes can potentially be used for the treatment of bone disorders.

\section{Results \\ Generation of mice with osteoblastic inactivation of PHDs leads to differential activation of HIF signaling}

Osteoblasts and osteocytes reside in the bone marrow, where there exists both heterogeneous areas of vascularization and oxygen tension (Regan et al. 2014; Spencer et al. 2014). In support of these findings, we used pimonidazole (PIMO) staining to detect microregional levels of functional hypoxia and found that within whole-bone sections of 8-wk-old mice, strong signals were detected within both endosteal osteoblasts and osteocytes (Fig. 1A). Compared with staining of the kidney, where nearly all tubular cells in the renal medullary cells stain positively for PIMO (Fig. 1B), quantification of PIMO-stained cells in the bone revealed $72 \%$ of osteocytes and $79 \%$ of endosteal osteoblasts stained for PIMO, demonstrating heterogeneous oxygen tension within the osteoblastic niche (Fig. 1C). Given these findings, we next sought to determine whether the PHD isoenzymes, which act as the oxygen sensors of the HIF signaling pathway, were expressed in bone cells. Primary osteoblasts were isolated from mice at postnatal days 0 and 7 and from 4-wk-old mice. Real-time PCR analysis confirmed the presence of bone-specific proteins, and Western blot analysis of whole-cell lysates revealed the presence of all three PHDs isoforms at all age points (Fig. 1D,E). Given these findings, we sought to identify the role of the PHD enzymes in regulating in vivo osteoblastic HIF signaling, osteoblast function, and bone homeostasis.

For this purpose, we systematically interrogated the individual and collective function of the three PHD isoenzymes by generating homozygous mice for the following conditional alleles: $P h d 1^{f l / f 1}, P h d 2^{f l / f 1}$, and $P h d 3^{f l / f 1}$ (single); Phd1 ${ }^{f 1 / f 1}$; Phd2 $2^{f 1 / f 1}$, Phd ' $2^{f 1 / f 1}$; Phd $3^{f 1 / f 1}$, and Phd1 $1^{f 1 / f 1}$; $P h d 3^{f l / f 1}$ (double); and $P h d 1^{f l / f 1}$; Phd2 ${ }^{f l / f 1}$; Phd3 ${ }^{f l / f 1}$ (triple). These mice were then crossed to mice expressing Crerecombinase under the control of the osterix (OSX) promoter to generate OSX-Cre; Phd1 ${ }^{f 1 / f 1}$, OSX-Cre; Phd2 ${ }^{f 1 / f 1}$,

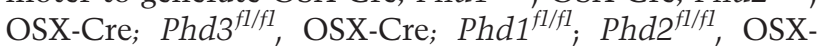
Cre; Phd $2^{f 1 / f 1}$; Phd $3^{f 1 / f 1}$, OSX-Cre; Phd1 $1^{f 1 / f 1} ; P h d 3^{f 1 / f 1}$, and OSX-Cre; Phd1 ${ }^{f l / f 1}$; Phd2 ${ }^{f l / f 1}$; Phd ${ }^{f l / f 1}$ mutant mice. OSXCre is a well-characterized transgene that mediates efficient Cre-recombinase activity in cells of the osteoblast lineage (Rodda and McMahon 2006). PHD activity is lost in osteoprogenitors upon Cre-mediated LOX-P excision of essential of $\mathrm{Fe}^{2+}$-binding residues within exon 2 of 
A

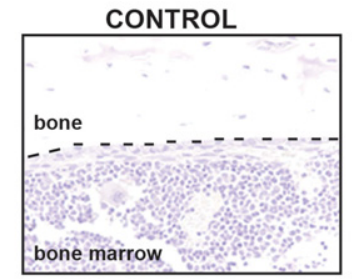

C

$\mathbf{F}$
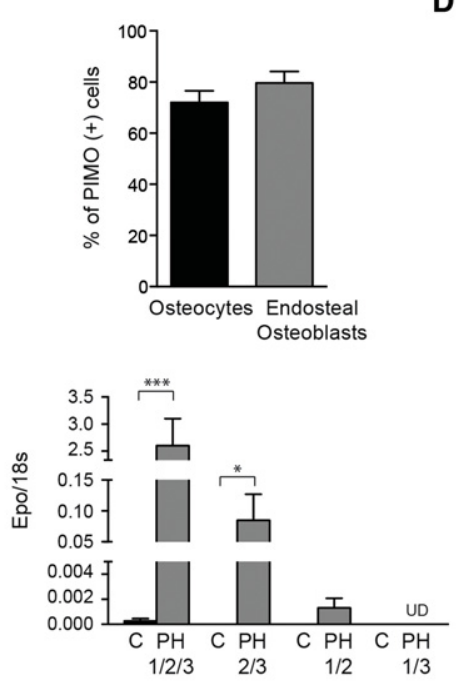

PIMO

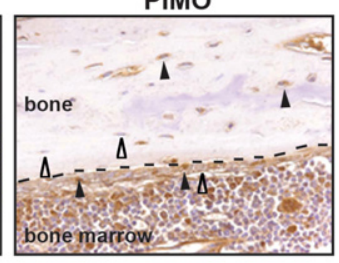

B

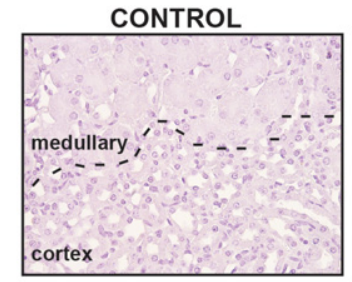

PIMO

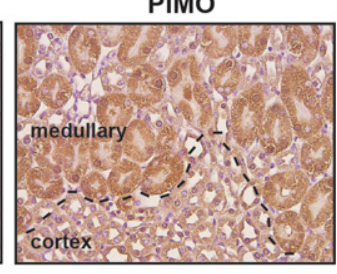

E
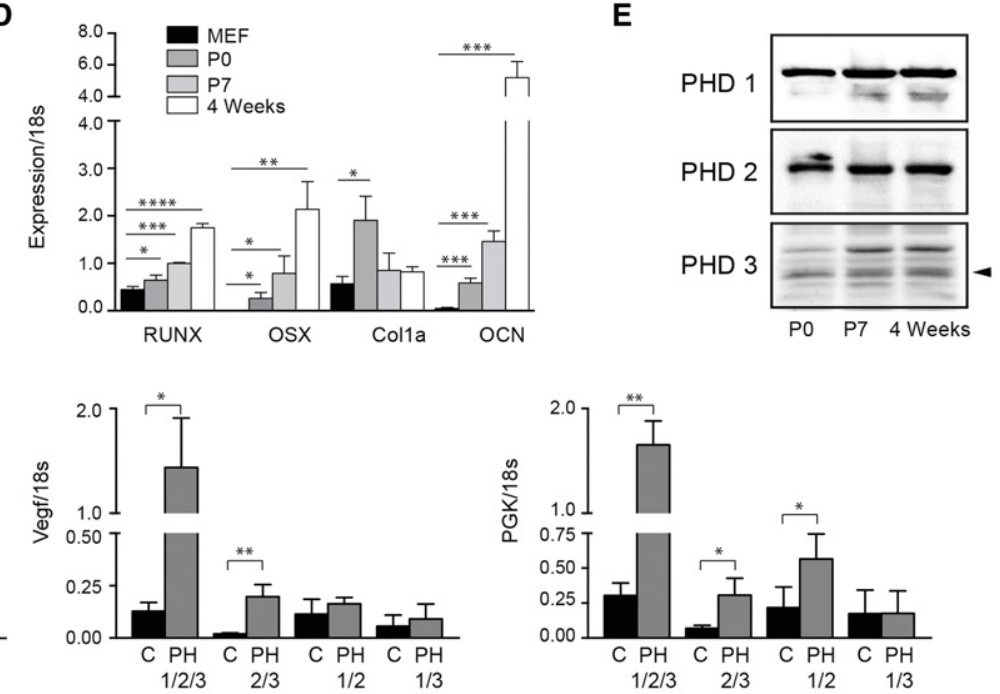

H
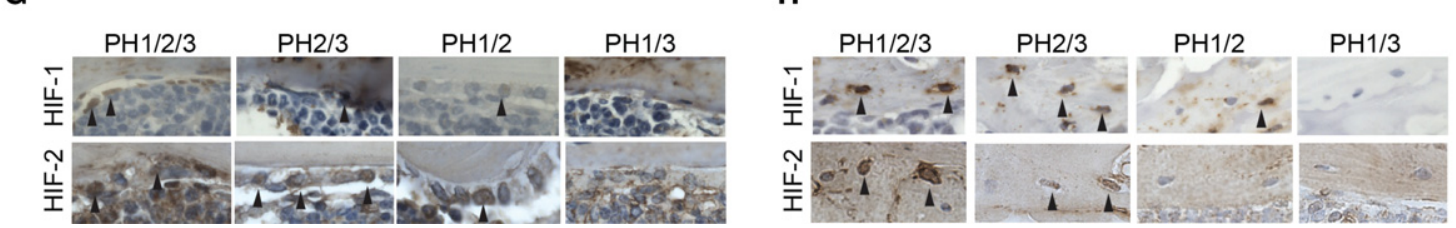

Figure 1. Differential regulation of osteoblastic HIF signaling through selective inactivation of PHDs. $(A)$ Representative PIMO staining of tibial sections of mice at $8 \mathrm{wk}$ of age. Arrowheads point to osteoblasts at the endosteal bone surface and osteocytes. Positively stained cells are marked with black arrowheads, and negative cells are marked with open arrowheads. (B) PIMO staining of a mouse kidney. (C) Quantification of PIMO-stained osteocytes and endosteal osteoblasts form tibia isolated from a wild-type mouse. $(D)$ Real-time PCR analysis of runx2 (RUNX), osterix (OSX), collagen 1 $\alpha$ (Colla), and osteocalcin (OCN) mRNA expression in calvarial osteoblasts isolated from mice at postnatal days 0 and 7 and at $4 \mathrm{wk}$ of age. $(E)$ Western blot analysis of whole-cell lysates isolated from calvarial osteoblasts at postnatal days 0 and 7 and at 4 wk of age. $(F)$ Real-time PCR analysis of EPO, VEGF, and PGK mRNA expression in bone tissues isolated from littermate control mice $(C)$ and OSX-Cre mutant mice. For each group analyzed, $n=4$. (UD) Undetectable levels. $(G, H)$ Immunohistochemical analysis of HIF-1 $\alpha(G$, top $)$ and HIF-2 $\alpha(G$, bottom) expression in osteoblasts lining the endosteal surface and in osteocytes $(H)$ in OSX-Cre mutant tibias. Arrowheads point to osteoblasts and osteocytes expressing HIF-1 $\alpha$ or HIF-2a.

each PHD isoform (Takeda et al. 2006, 2008). Genomic PCR analysis confirmed efficient and specific targeted deletion, as noted by the presence of the recombined $($ lox $-P)$ allele in bone tissue from mutants expressing Cre-recombinase (Supplemental Fig. S1A,B).

PHD enzymes regulate HIF protein stability through proline hydroxylation, which mediates pVHL binding and subsequent ubiquitin-mediated protein degradation. Loss of PHD activity results in increased HIF stability and the subsequent transactivation of HIF target genes (Semenza 2001). Real-time PCR analysis of whole-bone homogenates revealed that genetic inactivation of some but not all combinations of PHD isoenzymes in OSXexpressing cells resulted in the up-regulation of the HIF target genes erythropoietin (Epo), vascular endothelial growth factor (Vegf), and phosphoglycerate kinase (Pgk) (Fig. 1F). Osteoblastic loss of all three PHD isoforms resulted in the highest increase in the mRNA expression of HIF target genes, whereas combined loss of PHD2 and PHD3 and combined loss of PHD1 and PHD2 resulted in a modest increase of Epo, Vegf, and Pgk expression. In contrast, loss of PHD1 and PHD3 had no effect on HIF target gene expression. Consistent with these findings, immunohistological analysis revealed increased intensity of nuclear HIF- $1 \alpha$ and HIF- $2 \alpha$ staining in the osteoblasts and osteocytes of OSX-Cre; PHD $1^{f 1 / f 1} ;$ PHD2 $2^{f 1 / f 1} ; P H D 3^{f 1 / f 1}$, OSX-Cre; PHD2 $2^{f l / f 1} ;$ PHD $3^{f l / f 1}$, and OSX-Cre; PHD $1^{f l / f I^{\prime}}$; $P H D 2^{f l / f 1}$ mutant mice but not OSX-Cre; $P H D 1^{f l / f f^{\prime}}$; $P H D 3^{f l / f l}$ mutant mice (Fig. 1G,H). Thus, differential activation of HIF signaling can be achieved in bone tissue 
through the selective inactivation of specific PHD isoform combinations.

Selective inactivation of PHD modulates HIF signaling dosage to differentially regulate bone and hematopoietic homeostasis

Intriguingly, histological and $\mu \mathrm{CT}$ analysis of the OSX-Cre PHD mutant mice revealed increases in trabecular bone volume that were positively correlated with active HIF signaling, with the most severe phenotypes occurring in mice with the highest levels of HIF transcriptional activity. Specifically, examination of 12 -wk-old OSX-Cre; $P h d 1^{f l / f l} ; P h d 2^{f l / f l}$; $P h d 3^{f l / f l}$ femurs revealed an excessive increase in trabeculae in the metaphyseal and diaphyseal regions of the long bones when compared with their sexand age-matched littermate controls (Fig. 2A,B; Supplemental Table S1). These microarchitectural changes were associated with diminished bone marrow cellularity and trabecularization of the cortical bone, as marked by the replacement of cortical bone by trabecular-like porous bone structures (Fig. 2B). In support of these findings, $\mu \mathrm{CT}$ analysis of the mid-shaft of the femur revealed that average cortical thickness was substantially lower in OSXCre; $P h d 1^{f l / f 1}$; Phd $2^{f l / f 1}$; Phd $3^{f l / f 1}$ mutants when compared with age- and sex-matched littermate controls (Fig. 2D; Supplemental Table S3). In contrast, combined osteoblastic inactivation of $P h d 1$ with $P h d 2$ and $P h d 2$ with $P h d 3$ led to a significant increase in trabecular bone volume as a consequence of increased trabecular numbers. Importantly, these changes were not associated with excessive ossification of the long bones (Fig. 2A-C; Supplemental Table S1). In addition, $\mu \mathrm{CT}$ analysis revealed that loss of any combination of dual PHD isoforms did not significantly alter the average cortical bone thickness between OSX-PHD mutants and their sex- and age-matched
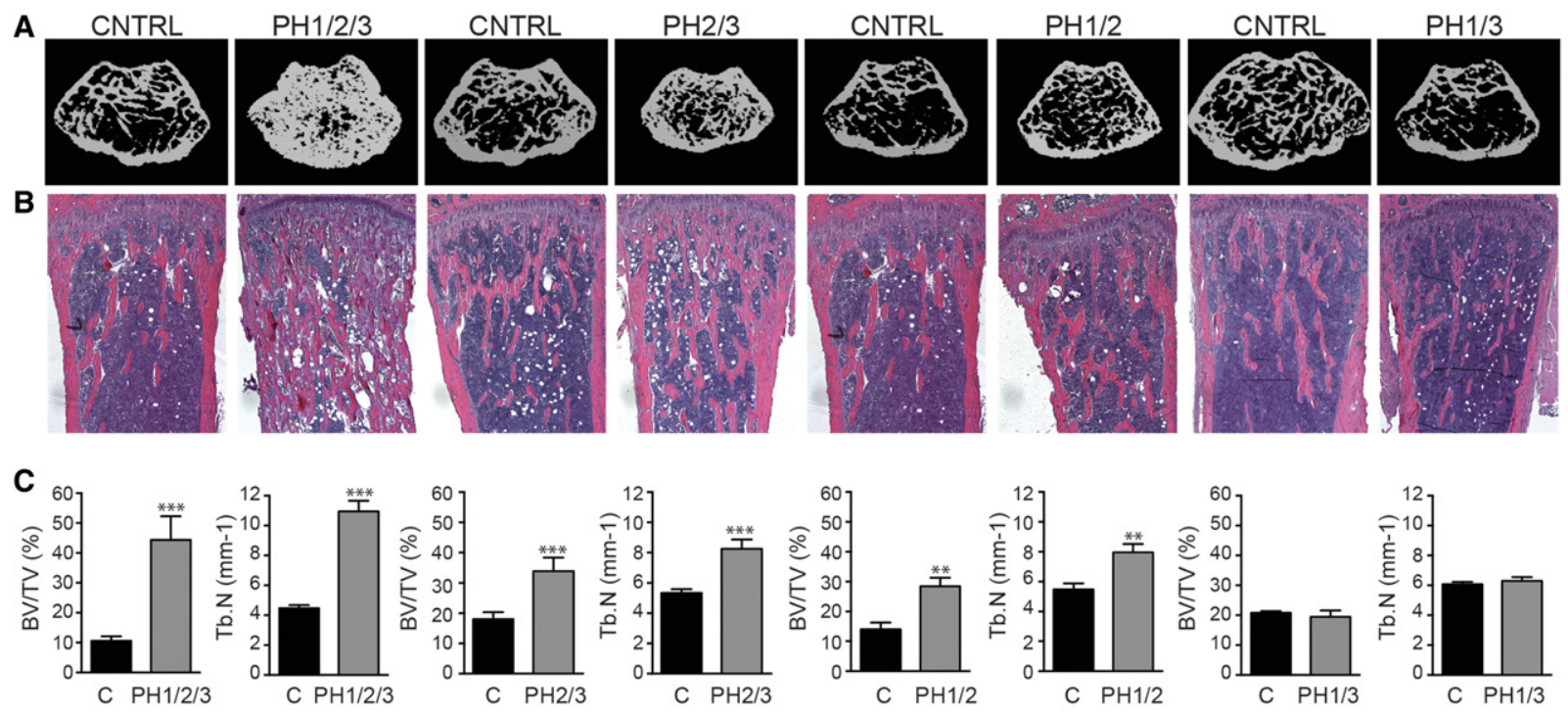

D
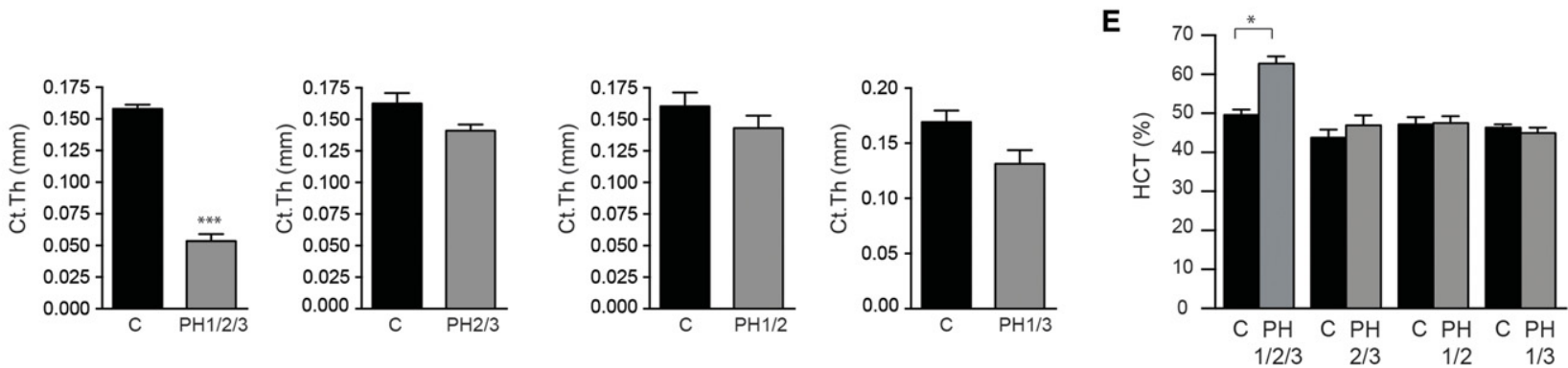

Figure 2. Selective inactivation of specific PHD isoform combinations increases trabecular bone volume without disrupting blood homeostasis. (A) Representative $\mu \mathrm{CT}$ images of distal femurs isolated from 12-wk-old control (CNTRL) and OSX-Cre mutant mice (axial view of metaphyseal region). (B) Representative images of H\&E-stained proximal tibias isolated from 12-wk-old control (CNTRL) and OSX-Cre mutant mice. $(C) \mu \mathrm{CT}$ analysis of trabecular bone volume (BV/TV) and trabecular number (Tb.N) from 12-wk-old control (C) and OSX-Cre mutant mice. For each group analyzed, OSX-Cre; $P h d 1^{f 1 / f 1} ; P h d 2^{f 1 / f 1} ; P h d 3^{f l / f 1}(\mathrm{PH} 1 / 2 / 3), n=6 ; \mathrm{OSX}-\mathrm{Cre} ; P h d 2^{f 1 / f 1} ; P h d 3^{f 1 / f 1}$ $(\mathrm{PH} 2 / 3), n=8$; OSX-Cre; Phd1 ${ }^{f 1 / f 1}$; $P h d 2^{f 1 / f 1}(\mathrm{PH1} / 2), n=7$; and OSX-Cre; $P h d 1^{f 1 / f 1}$; $P h d 3^{f 1 / f 1}(\mathrm{PH} 1 / 3), n=7$. (D) $\mu \mathrm{CT}$ analysis of cortical bone thickness (Ct.Th) of 12-wk-old control (C) and OSX-Cre mutant mice. For each group analyzed, OSX-Cre; Phd1 ${ }^{f 1 / f 1}$; Phd2 $2^{f l / f 1}$;

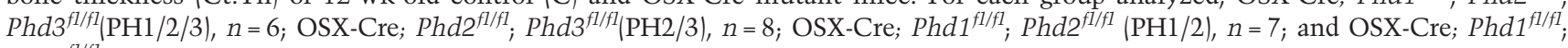
$P h d 3^{f l / f 1}(\mathrm{PH} 1 / 3), n=4$. (E) Percentage of hematocrit $(\mathrm{HCT})$ in control (C) and OSX-Cre mutant mice at 12 wk of age. For each group an-

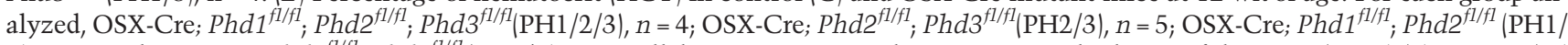
$2), n=5$; and OSX-Cre; $P h d 1^{f 1 / f 1} ; P h d 3^{f l / f 1}(\mathrm{PH1} / 3), n=5$. All data are represented as mean \pm standard error of the mean $\left.(\mathrm{SEM}) .(*) P<0.05 ;{ }^{* *}\right)$ $P<0.01 ;\left(^{* * *}\right) P<0.001$, determined by Student's $t$-test. 
littermate controls (Fig. 2D; Supplemental Table S3). Importantly, histological and $\mu \mathrm{CT}$ analysis revealed no differences in bone volume, trabecular number, trabecular thickness, or trabecular separation between OSX-Cre; $P h d 1^{f l / f 1}$; Phd ${ }^{f l / f 1}$, OSX-Cre; Phd1 ${ }^{f l / f 1}$, OSX-Cre; Phd2 ${ }^{f l / f 1}$, or OSX-Cre; $P h d 3^{f 1 / f 1}$ and their sex- and age-matched littermate controls (Fig. 2A-C; Supplemental Fig. S2; Supplemental Table S2). These findings demonstrate that selective inactivation of specific PHD isoform combinations differentially regulates trabecular but not cortical bone homeostasis by modulating HIF signaling dosage.

The osteoblastic niche plays an important role in regulating multiple hematopoietic lineages (Wu et al. 2009). We previously demonstrated that genetic loss of either pVHL or all three PHD isoforms in cells of the osteoblast lineage augments HIF signaling and modulates erythropoiesis by directly regulating the production of EPO within the local bone marrow microenvironment. Osteoblastic EPO production led to the expansion of the erythroid lineage, resulting in the development of polycythemia as marked by a significant increase in hematocrit and red blood cell (RBC) numbers (Rankin et al. 2012). Given these observations, we sought to determine whether selective inactivation of dual PHD isoform combinations would also alter mature hematopoietic linages. To address this question, we compared cells of the myeloid and lymphoid lineages from peripheral blood isolated from 12-wk-old OSX-Cre; Phd1 ${ }^{f 1 / f 1}$; Phd $2^{f 1 / f 1}$; Phd ${ }^{f 1 / f 1}$, OSX-Cre; Phd1 ${ }^{f 1 / f 1}$; Phd2 $2^{f 1 / f 1}$, OSX-Cre; Phd2 ${ }^{f 1 / f 1} ; P h d 3^{\prime}{ }^{f 1 / f 1}$, and OSX-Cre; $P h d 1^{f l / f 1}{ }^{\prime} P h d 3^{f l / f 1}$ mutant mice. In support of our previous work, genetic inactivation of all PHD isoforms significantly elevated RBCs and hematocrit while diminishing lymphocyte numbers (Fig. 2E; Supplemental Table S4). In contrast, despite elevation of the HIF target gene Epo and the expansion of the osteoblastic compartment of the hematopoietic niche, selective inactivation of dual PHD isoform combinations did not alter hematopoietic homeostasis in OSX-PHD mutant mice when compared with controls. Both mature myeloid and lymphoid blood lineages in OSX-Cre; Phd1 ${ }^{f 1 / f 1}$; Phd $2^{f 1 / f 1}$, OSX-Cre; Phd2 $2^{f l /}$ ${ }^{f 1}$; $P h d 3^{f l / f 1}$, and OSX-Cre; $P h d 1^{f 1 / f 1}$; $P h d 3^{f 1 / f 1}$ mice were indistinguishable from age- and sex-matched littermate control mice (Supplemental Table S4). Importantly, this demonstrates that changes in bone homeostasis can be achieved through both collective and selective inhibition of the PHD isoforms; however, functional changes in hematopoiesis require the loss of all three PHD isoforms within the local bone marrow microenvironment.

\section{Osteogenic-angiogenic coupling is not required for HIF-mediated changes in bone homeostasis}

The relationship between angiogenesis and osteogenesis is highlighted by lineage tracing studies demonstrating that, during endochondral ossification, osteoblast precursors reside in close spatial and temporal association with capillary bed invasion (Maes et al. 2010a). This coupling of osteogenesis to angiogenesis is mediated in part by HIF signaling (Wang et al. 2007; Schipani et al. 2009). In support of this, we found that genetic inactivation of all three PHD isoforms led to severe HIF activation marked in part by the up-regulation of VEGF mRNA (Fig. 1F). This was associated with a stark increase in trabecular bone and hypervascularization, as demonstrated by CD31 immunohistochemistry (Fig. 3A). Surprisingly, despite the increased trabecular bone volume seen in OSX-Cre; $P h d 2^{f 1 / f 1} ; P h d 3^{f l / f 1}$ and OSX-Cre; Phd1 ${ }^{f 1 / f 1} ; P h d 2^{f 1 / f 1}$ mice, CD31 staining showed no differences in microvascular density between OSX-Cre; $P h d 2^{f 1 / f 1}$; $P h d 3^{f 1 / f 1}$ and $P h d 1^{f 1 / f 1}$; $P h d 2^{f l / f 1}$ mutant mice when compared with their littermate controls (Fig. 3A). In support of this, quantification of vasculature within the metaphyseal and diaphyseal regions of whole-bone sections isolated from 12 -wk-old mice revealed indistinguishable numbers of blood vessels between OSX-Cre; Phd2 $2^{f l / f 1}$; Phd ${ }^{f l / f 1}$, OSX-Cre; Phd1 ${ }^{f l / f 1}$; $P h d 2^{f l / f 1}$, and littermate controls (Fig. 3B). Recent reports demonstrate that CD $31^{\text {high }} /$ endomucin ${ }^{\text {high }}$ blood vessels are associated with coupling osteogenesis to angiogenesis (Kusumbe et al. 2014). Serial sections of tibias isolated from 8-wk-old OSX-Cre; Phd2 ${ }^{f l / f 1} ; P h d 3^{f l / f 1}$ mutants and littermate control mice were stained for either CD31 or endomucin (Fig. 3C). Quantification of metaphyseal and diaphyseal regions of blood vessels staining for both markers revealed no differences in the number of CD31 $1^{\text {high }}$ endomucin ${ }^{\text {high }}$ blood vessels between mutants and littermate controls (Fig. 3C). In addition, only a modest increase in VEGF mRNA expression was detected between OSX-Cre; Phd $2^{f l / f 1} ; P h d 3^{f l / f 1}$ and their controls, and no differences were detected between the OSX-Cre; Phd1 ${ }^{f l / f 1}$; Phd $2^{f l / f 1}$ mutant and control mice (Fig. 1F). Furthermore, ELISAs revealed that circulating levels of VEGF in serum remained unaffected by genetic inactivation of PHD isoenzymes (Fig. 3B). These data suggest that PHD/HIF signaling can regulate bone homeostasis independently of HIF-mediated neoangiogenesis.

\section{Genetic inactivation of osteoblastic Phd2 and Phd3 inhibits osteoblast-mediated osteoclastogenesis through the modulation of OPG}

Our findings detailed above led us to the unexpected conclusion that the PHD oxygen sensors could modulate bone homeostasis independent of HIF-mediated angiogenesis. We therefore considered the possibility that PHD/ HIF signaling could regulate bone homeostasis by a cellautonomous mechanism. Given that combined genetic inactivation of Phd2 and Phd3 resulted in the most striking increase in trabecular bone volume that was not associated with increased VEGF production or enhanced bone microvasculature density, we analyzed the osteoclast compartment in OSX-Cre; Phd2 ${ }^{f 1 / f 1}$; Phd $3^{\text {fl/fl }}$ mutant mice to determine the contribution that these PHD enzymes had on bone resorption. Alcian blue histological staining of proximal tibias revealed increased cartilage remnants in the trabeculae of 12 -wk-old OSX-Cre; $P h d 2^{f l / f 1} ; P h d 3^{f l / f 1}$ mice when compared with littermate controls, indicating impaired remodeling of the primary spongiosa (Fig. 3D). Quantitative histomorphometry of tartrate-resistant acid phosphatase (TRAP)-positive cells showed a marked reduction in TRAP-positive 

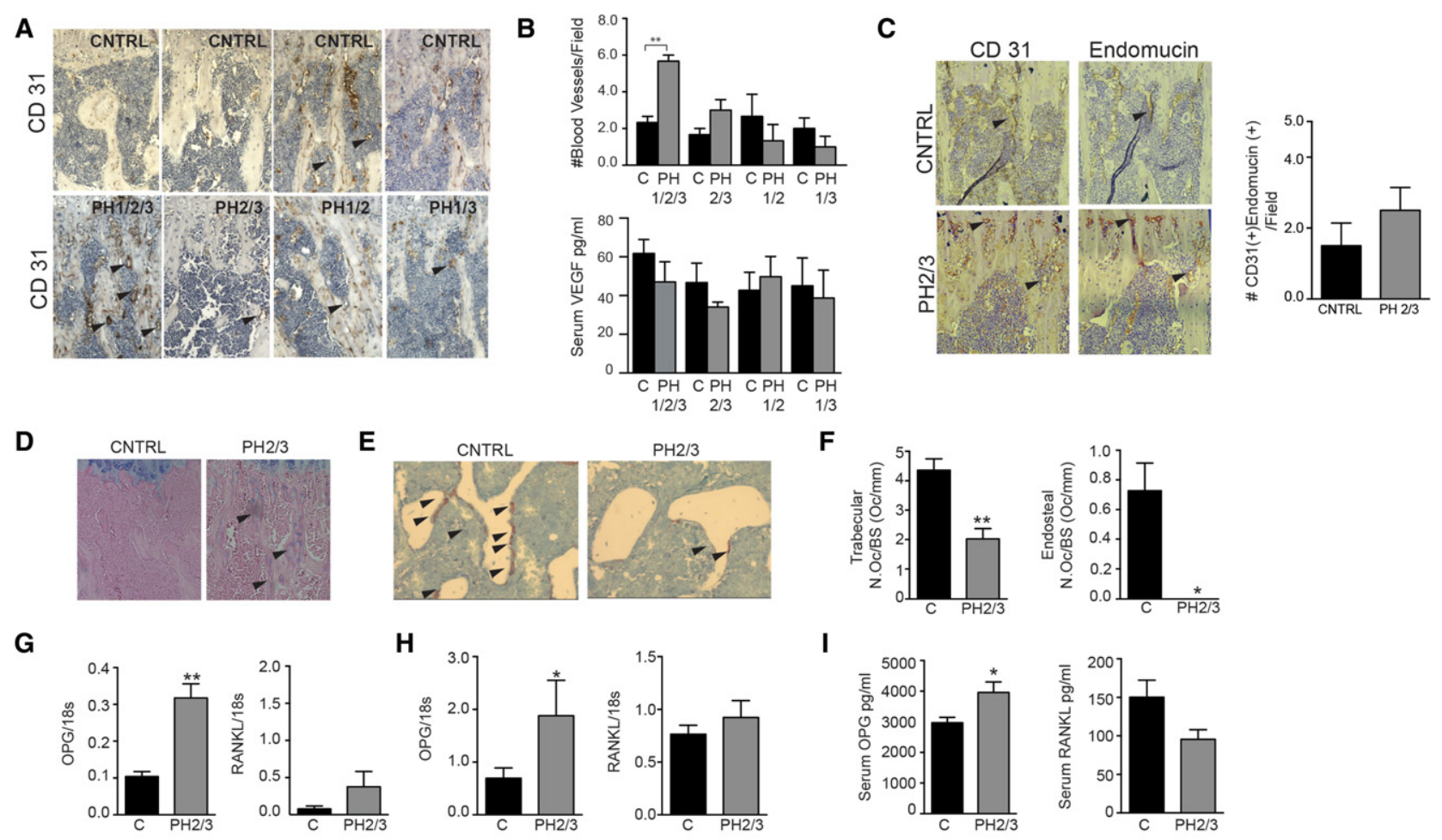

I
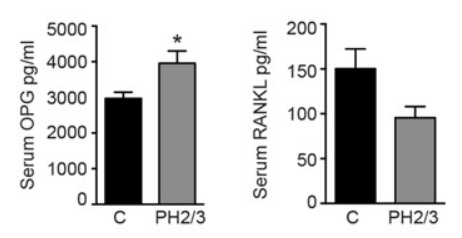

$\mathbf{J}$

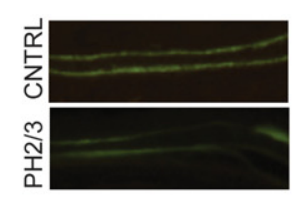

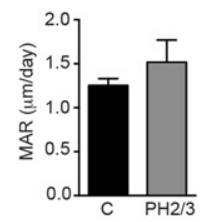

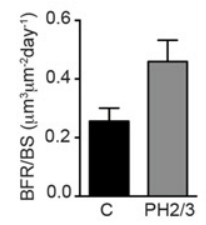

$\mathbf{K}$

L

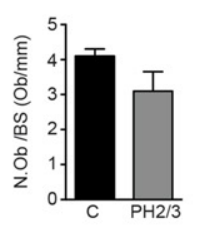

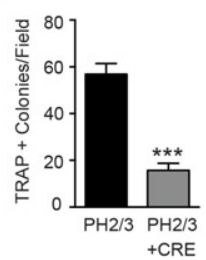

M

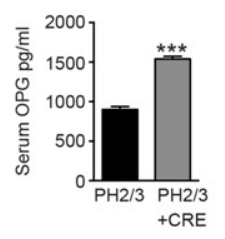

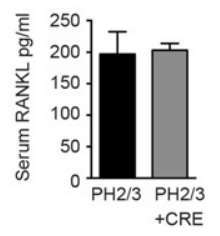

Figure 3. Osteoblastic inactivation of PHD2 and PHD3 inhibits osteoclastogenesis. $(A)$ Representative images of CD31 immunohistochemistry to visualize vascularization in control (CNTRL) and OSX-Cre PHD mutant mice. Blood vessels are indicated with black arrowheads. $(B$, top $)$ Quantification of blood vessels in bones in 12-wk-old control and OSX-Cre PHD mutant mice. (Bottom) Analysis of VEGF protein levels in the serum of 12-wk-old control (C) and OSX-Cre PHD mutant mice. $(C$, left) Representative images of serial sections stained for CD31 and endomucin. Colocalized staining of blood vessels is indicated with black arrowheads. (Right) Quantification of $\mathrm{CD} 31^{\text {high }} /$ endomucin ${ }^{\text {high }}$ blood vessels in 8-wk-old control and mutant mice. $(D)$ Representative images of Alcian blue-stained control (CNTRL) and OSX-Cre; $P h d 2^{f 1 / f 1}$; $P h d 3^{f 1 / f 1}(\mathrm{PH} 2 / 3)$ mutant proximal tibias isolated from 12-wk-old mice. The arrowheads point to unremodeled cartilage. (E) Representative images of tartrate-resistant acid phosphatase (TRAP)-stained proximal tibias isolated from 12-wkold control and mutant mice. The arrowheads point to TRAP-positive osteoclasts. $(F)$ Quantification of TRAP-positive multinucleated osteoclasts in proximal tibias. The number of trabecular and endosteal osteoclasts per bone surface (trabecular N.Oc./BS, $n=6$; endosteal N.Oc/BS, $n=4$ ) is shown. ( $G$ ) Real-time PCR analysis of OPG and RANKL mRNA in bone tissues isolated from 12-wk-old mice. For each group analyzed, $n=4$. $(H)$ Real-time PCR analysis of OPG and RANKL mRNA isolated from primary calvarial cultures. For each group analyzed, $n=4$. (I) Analysis of OPG and RANKL protein levels in the serum of 12-wk-old mice as determined by ELISA. For each group analyzed, $n=4$. (J, left) Representative image of dual calcein-labeled femurs from 12-wk-old mice. (Right) Assessment of the mineral apposition rate (MAR) and bone formation rate per bone surface (BFR/BS) by quantification of dual calcein-labeled sections of distal femur from 12-wk-old mice. For each group analyzed, $n=6 .(K)$ Quantification of trabecular osteoblast numbers per bone surface $(n=3)$. $(L)$ Quantification of TRAP-positive colonies from cocultures of wild-type bone marrow stromal cells and primary osteoblast cultures isolated from PHD2 ${ }^{\mathrm{fl} / \mathrm{fl}}$; PHD $3^{\mathrm{fl} / \mathrm{fl}}$ treated with either control or adeno-CRE virus $(n=3)$. $(M)$ Analysis of secreted OPG and RANKL protein levels from coculture experiments as determined by ELISA $(n=3)$. All data are represented as mean \pm standard error of the mean (SEM). (*) $P<0.05 ;\left(^{* *}\right) P<0.01 ;\left(^{* * *}\right) \mathrm{P}<0.001$, determined by Student's $t$-test.

multinucleated trabecular and endosteal osteoclasts in OSX-Cre; $P h d 2^{f 1 / f 1}$; Phd ${ }^{f l / f 1}$ mutant mice (Fig. 3E,F).

The RANK/RANKL/OPG axis contributes to bone homeostasis by facilitating osteoblast-mediated osteoclastogenesis, a major element of proper bone remodeling (Boyle et al. 2003). Given the reduction in osteoclast numbers, we next examined the OPG/RANK/RANKL signaling pathway in our mutant mice. In support of our static histomorphometic data, molecular analysis of whole-bone homogenates isolated from OSX-Cre; $P h d 2^{f l / f 1} ; P h d 3^{f l / f 1}$ mice revealed increased expression of OPG but not RANKL mRNA levels when compared with control 
mice (Fig. 3G). Because total bone preparations contain many cell types, we also directly assessed whether mutant osteoblasts from OSX-Cre; Phd $2^{f 1 / f 1}$; Phd $3^{f l / f 1}$ produced more OPG than control osteoblasts. Notably, cultured primary calvarial cultures isolated from OSXCre; Phd2 $2^{f l / f 1}$; Phd ${ }^{f l / f 1}$ mice showed increased expression of OPG but not RANKL mRNAs levels (Fig. 3H). Accordingly, these mutant mice also had increased OPG but not RANKL serum levels when compared with littermate controls (Fig. 3I). We next cocultured primary osteoblasts isolated from the calvaria of $P h d 2^{f 1 / f l f l} ; P h d 3^{f 1 / f 1}$ mice with wild-type bone marrow stromal cells. We found that in vitro, genetic inactivation of PHD2 and PHD3 resulted in diminished formation of TRAP-stained cells when compared with controls (Fig. 3L). In addition, we found increased secretion of OPG but not RANKL from the media collected from cocultures of osteoblasts harboring genetically inactivated PHD2 and PHD3 when compared with controls (Fig. 3M).

Dynamic histomorphometry revealed no differences in mineral apposition rates (MARs), bone formation rates, or osteoblast numbers between OSX-Cre; Phd2 ${ }^{f l / f 1} ; P h d 3^{f 1 / f 1}$ mice and littermate controls, suggesting that bone formation rates were similar between the two groups of mice (Fig. 3J,K). In support of our in vivo findings, in vitro primary calvarial cells isolated from OSX-Cre; Phd $2^{f 1 / f 1}$; $P h d 3^{f l / f 1}$ mice cultured under osteogenic conditions for $14 \mathrm{~d}$ showed no differences in alkaline phosphatase (ALP) activity or the expression of osteoblastic marker genes Colla and BSP, indicating that osteoblastic loss of both PHD2 and PHD3 did not promote changes in osteoblast differentiation (Supplemental Fig. S3A,B). Taken together, we demonstrate that PHD2 and PHD3 influence bone homeostasis through the modulation of OPG to suppress osteoblast-mediated osteoclastogenesis.

\section{HIF signaling is both necessary and sufficient to inhibit osteoclastogenesis and bone resorption through the modulation of OPG}

We next sought to elucidate the molecular mechanism modulating the expression of OPG within our PHD mutant mice. To determine whether osteoblasts express OPG in a HIF-dependent manner, we cultured the primary osteoblast cell line MC3T3 E1 in either 21\% oxygen (normoxia) or $0.5 \%$ oxygen (hypoxia) to induce HIF signaling in vitro. In response to hypoxia, we saw an increase in mRNA expression of the HIF target gene Vegf as well as a twofold induction of OPG mRNA levels, indicating that HIF signaling drives OPG expression under physiologic conditions (Fig. 4A).

To access the contribution of HIF-1 $\alpha$ and HIF-2 $\alpha$ in the modulation of OPG, MC3T3 E1 cells were transiently transfected with HIF variants in which both prolyl hydroxylation sites were replaced by alanine. These variants cannot be hydroxylated by PHDs and are therefore constitutively stabilized regardless of oxygen tension (Kondo et al. 2002). Expression of both nondegradable HIF-1a and HIF-2 $\alpha$ variants resulted in augmented HIF activity, as demonstrated by the induction of the HIF target gene
Vegf (Supplemental Fig. S4A). However, statistically significant increases in OPG mRNA expression were seen only in cells expressing constitutively active HIF-2a (Fig. 4B). Notably, as only a moderate induction of VEGF mRNA was seen in cells expressing constitutively active HIF-1 $\alpha$, we could not dismiss the possibility that HIF-1a also has the potential to direct the regulation of OPG (Supplemental Fig. S4A).

HIF transcriptional activity can modulate gene expression through direct and indirect mechanisms. To determine whether OPG is a direct target of HIF-2a, we searched the Tnfrsf11B (OPG) promoter for consensus hypoxia-responsive element (HREs) containing a conserved RCGTG sequence. Promoter analysis revealed the presence of HREs within the Tnfrsf11B (OPG) promoter adjacent to the transcriptional start site. To evaluate HIF- $2 \alpha$ binding to HREs present in this region, we transfected MC3T3 E1 cells with a HA-tagged constitutively active variant of HIF-2 $\alpha$ (HA-HIF-2adPA). Western blot analysis of cell lysates confirmed the presence of the nondegradable HA-tagged HIF-2 $\alpha$ variant (Supplemental Fig. S4B). Chromatin immunoprecipitation (ChIP) analysis revealed that HIF-2 a directly bound to HREs in the Tnfrsf11B promoter, with binding exceeding that of HIF-2 $\alpha$ within the Vegf HRE promoter, an established HIF target gene (Fig. 4C). These data indicate that OPG is a direct HIF-2 $\alpha$ target gene.

Next, to directly interrogate the contribution of osteoblastic HIF signaling in mediating osteoclastogenesis and bone homeostasis, we bred OSX-Cre mice to transgenic mice in which conditional alleles of either the Hif-1a or Hif-2a (OSX-Cre; Hif1dPA and OSX-Cre; Hif2dPA) variants escape recognition of $\mathrm{pVHL}$, resulting in the constitutive activation of HIF signaling (Kim et al. 2006). Realtime PCR analysis of whole-bone homogenates revealed augmented HIF activity in both OSX-Cre; Hif1dPA and OSX-Cre; Hif2dPA mice. Isoform specificity was confirmed by examination of genes regulated by both HIF$1 \alpha$ and HIF-2 $\alpha$ (Vegf) or uniquely by HIF- $1 \alpha(P g k)$ or HIF$2 \alpha(E p o)$ (Supplemental Fig. S4C). Similar to the OSXCre; $P h d 2^{f l / f I}$; $P h d 3^{f l / f l}$ mice, OSX-Cre; Hif2dAP exhibited statistically significant increases in mRNA expression of OPG, an increased ratio of serum OPG to RANKL, diminished numbers of trabecular and endosteal osteoclasts, and no differences in osteoblast numbers, thereby phenocopying our OSX-Cre; Phd $2^{f l / f 1}$; Phd $3^{f l / f 1}$ mutant mice (Fig. 4D-H; Supplemental Fig. S4D,E). In support of these findings, histological staining (proximal tibia) and $\mu \mathrm{CT}$ analysis (distal femur) revealed increases in trabecular bone volume and trabecular numbers in OSX-Cre; Hif2dPA when compared with sex- and age-matched littermate controls (Fig. 4I). Quantification of $\mu \mathrm{CT}$ images confirmed a 2.5-fold increase in trabecular bone volume in the metaphyseal region associated with a significant increase in trabecular numbers in OSX-Cre; Hif2dPA (Fig. 4J). Importantly, while we did note induction of the HIF target gene Vegf in whole-bone homogenates isolated from OSXCre; Hif1dPA mice, demonstrating stabilization and transcriptional activation of HIF-1a, consistent with data reported by Kim et al. (2006), this induction was lower 
A

B
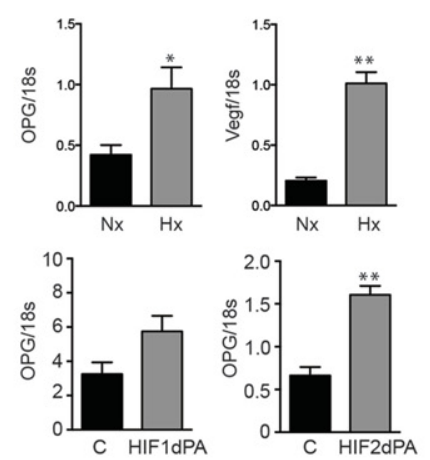

C

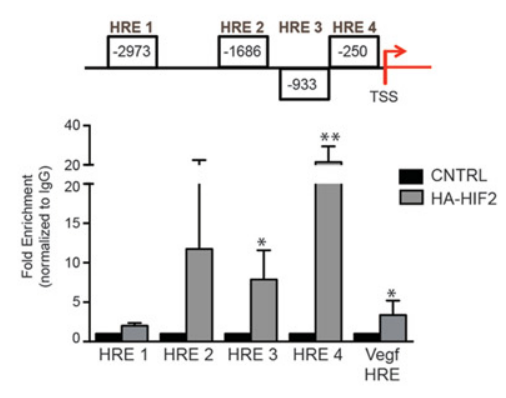

D

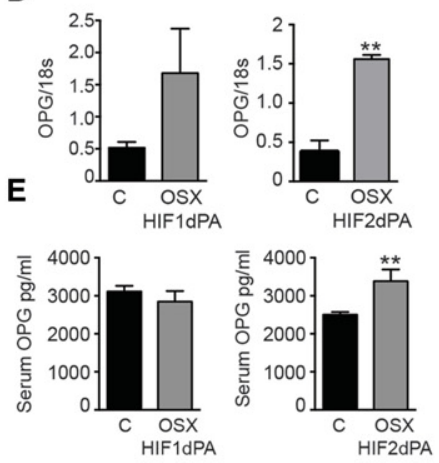

F
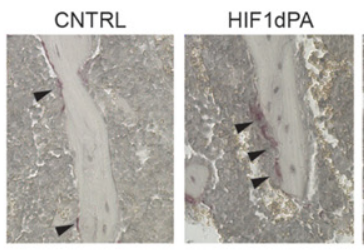
HIF1dPA

I
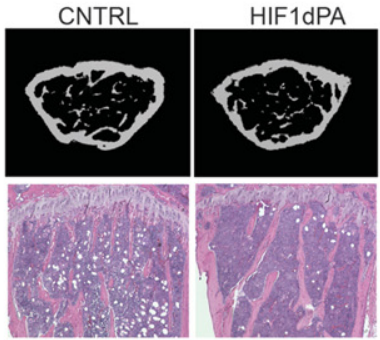

J

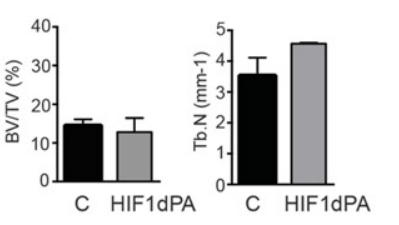

CNTRL
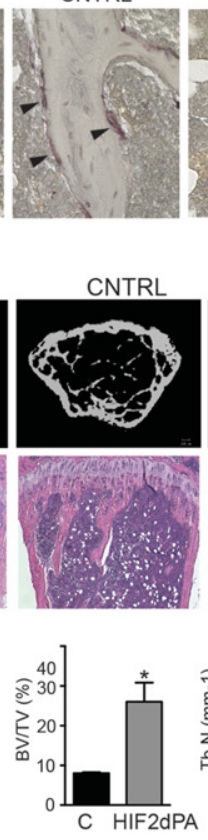

HIF2dPA

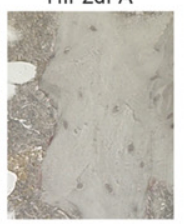

HIF2dPA
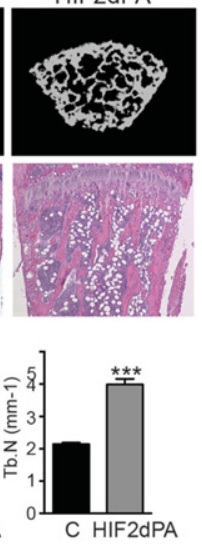

G

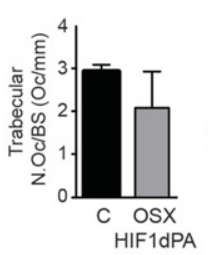

K
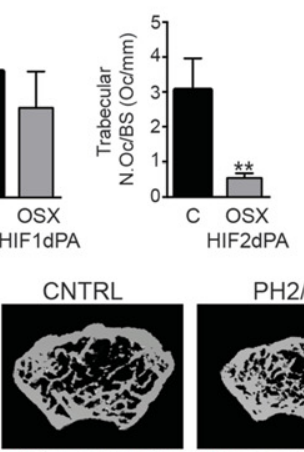

H

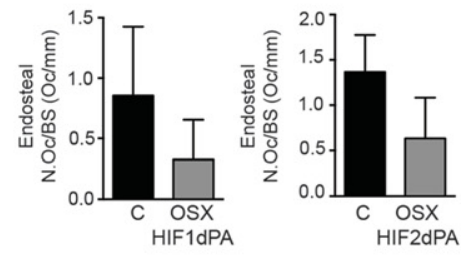

Figure 4. HIF regulates osteoblastic OPG to modulate osteoclastogenesis. (A) Real-time PCR analysis of OPG and VEGF expression in MC3T3 E1 cells exposed to $21 \%$ oxygen $(\mathrm{Nx})$ or $0.5 \%$ oxygen $(\mathrm{Hx})$. ( $B$ ) Real-time PCR analysis of OPG expression of MC3T3 E1 cells transfected with constitutively active variants of HIF-1 $\alpha$ (HIF-1dPA) or HIF-2 $\alpha$ (HIF-2dPA). (C, top) Schematic representation of the mouse Tnfrsf11b (OPG) promoter upstream of the transcriptional start site (TSS). Four potential HIF-binding sites (hypoxia-responsive element [HREs]) are indicated with white boxes. (Bottom) Chromatin immunoprecipitation (ChIP) assay analysis of HIF-2 binding to HREs located within the OPG promoter in MC3T3 E1 cells transfected with a nondegradable HA-tagged variant of HIF-2a. HIF-2a occupancy on the VEGF promoter HRE was used as the positive control. $(D)$ Real-time PCR analysis of OPG mRNA from whole-bone homogenates of 12-wk-old mice. For each group analyzed, $n=3$. (E) Analysis of OPG protein levels in the serum of 12-wk-old mice as determined by ELISA. For each group analyzed, $n=4$. $(F)$ Representative images of TRAP-stained proximal tibias isolated from 12-wk-old mice. $(G, H)$ Quantification of TRAP-positive multinucleated trabecular $(G ; n=5)$ and endosteal $(H ; n=3)$ osteoclasts in proximal tibias by analyzing the number of osteoclasts per bone surface (N.Oc./BS). (I, top) Representative $\mu \mathrm{CT}$ images of distal femurs (axial view of metaphyseal region) isolated from 12-wk-old mice. (Bottom) Representative images from H\&E staining of proximal tibias isolated from 12-wk-old mice. (J) $\mu \mathrm{CT}$ analysis of distal femurs of trabecular bone volume (BV/TV) and trabecular number (Tb.N) from 12-wk-old mice. (CNTRL) Control; (HIF1dPA) OSX-Cre; Hif1dPA; (HIF2dPA) OSX-Cre; HIf2dPA. (K, top) Representative $\mu C T$ images of distal femurs (axial view of metaphyseal region) isolated from 12-wk-old mice. (Bottom) Representative H\&E images of proximal tibias isolated from control (CNTRL) and

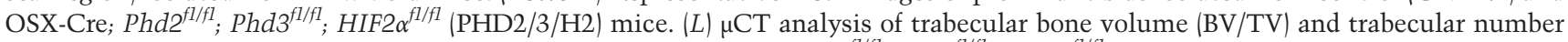

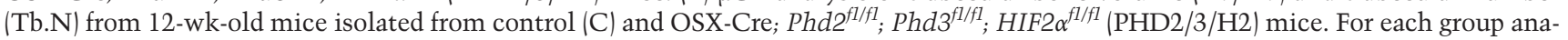
lyzed, $n=5$. All data are represented as mean \pm standard error of the mean (SEM). $\left.{ }^{*}\right) P<0.05$; $\left(^{* *}\right) P<0.01 ;(* * *) P<0.001$, determined by Student's $t$-test. Note: Data shown from CNTRL and PHD2/3 mice are taken from Figure 2.

than transcriptional activation by HIF-2 $\alpha$ (Supplemental Fig. S4C). As such, we could not dismiss the possibility that HIF-1a also has the potential to induce expression of OPG and modulate osteoclastogenesis. Taken together, our data indicate that augmented HIF signaling in osteoprogenitor cells is sufficient to alter bone homeostasis through the up-regulation of OPG and subsequent inhibition of osteoclastogenesis. 
Next, to determine whether HIF-2 $\alpha$ was necessary for PHD2- and PHD3-mediated changes in bone homeostasis, we conditionally inactivated HIF-2 $\alpha$ in OSX-Cre; Phd $2^{f 1 / f 1}$; $P h d 3^{f l / f 1}$ mice. $\mu \mathrm{CT}$ and histological analysis of OSX-

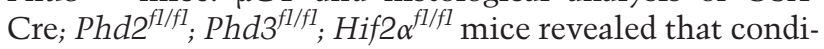
tional deletion of HIF- $2 \alpha$ resulted in a complete abrogation of the observed bone phenotype in OSX-Cre; Phd2 $2^{f 1 / f 1}$; $P h d 3^{f l / f l}$ (Fig. 4K). Quantification of $\mu \mathrm{CT}$ analysis confirmed these observations, demonstrating the normalization of bone volume and trabecular numbers (Fig. 4L). Examination and quantification of the osteoclast compartment revealed no significant differences in either TRAPstained osteoclasts or OPG and RANKL mRNA in OSXCre; Phd2 ${ }^{f l / f 1}$; Phd $3^{f 1 / f 1}$; HIF2 $\alpha^{f l / f 1}$ mice when compared with age- and sex-matched littermate controls (Supplemental Fig. S4G,H). Collectively, these data demonstrate that HIF-2 $\alpha$ is necessary within the context of combined PHD2 and PHD3 inactivation to inhibit bone resorption through osteoblastic modulation of OPG.

\section{Repression of endogenous HIF signaling enhances} osteoclastogenesis

To test the requirement for PHD/HIF signaling in modulating the physiologic expression of OPG, we used mice in which conditional alleles for HIF- $1 \alpha$ and HIF- $2 \alpha$ contain Lox-P sites flanking exon 2 (which encodes the DNA-binding domain), resulting in an out-of-frame deletion of exon 2 and the loss of HIF-1 $\alpha$ or HIF-2 $\alpha$ expression following Cre recombination (Ryan et al. 1998; Gruber et al. 2007). In vitro, inactivation of HIF-1 $\alpha$ and HIF- $2 \alpha$ was achieved by infecting primary osteoblasts isolated from these mice with adenovirus driving the expression of Cre-recombinase. In response to hypoxia, loss of either HIF-1 $\alpha$ or HIF-2a resulted in diminished mRNA expression of the HIF target gene $P g k$ (Fig. 5A,B). Interestingly, inactivation of HIF-2 $\alpha$ resulted in diminished OPG mRNA expression (Fig. 5B), indicating a requirement of HIF signaling for the regulation of osteoblastic OPG expression in response to hypoxia.
A

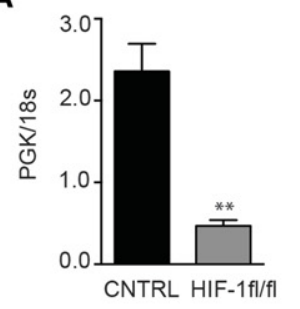

B

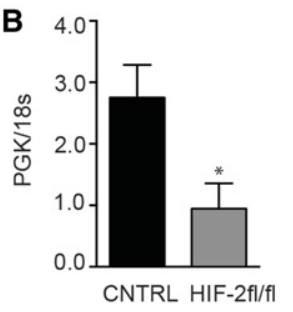

G

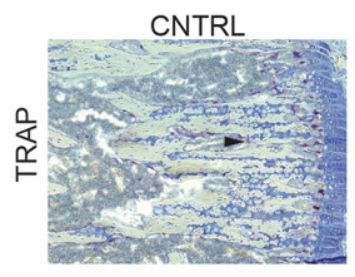

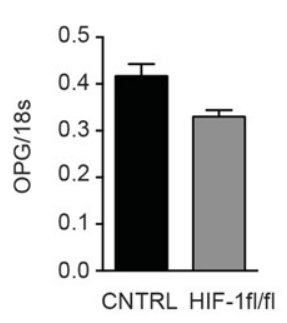

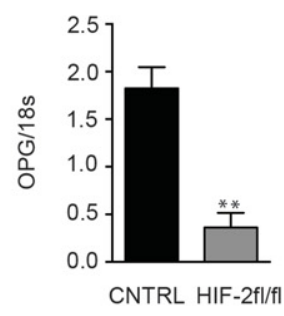

OSX-HIF1/HIF2

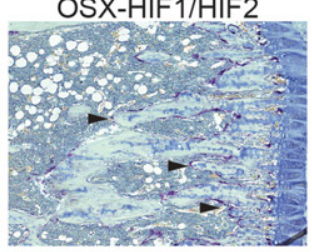

C

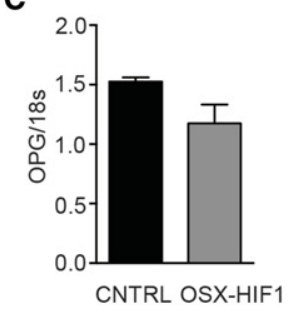

E

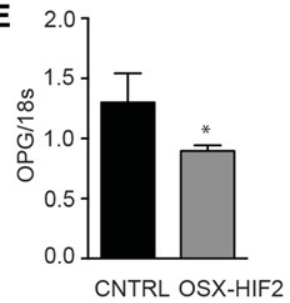

H

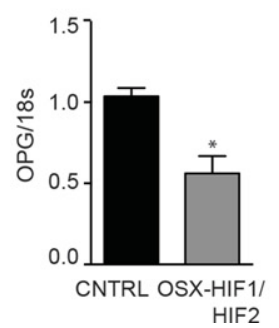

HIF2
D

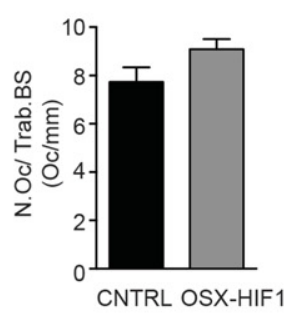

$\mathbf{F}$

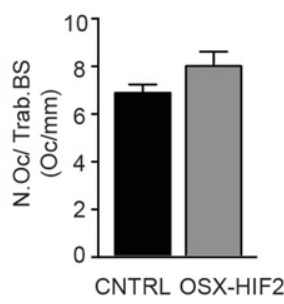

I

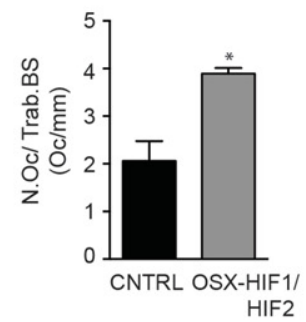

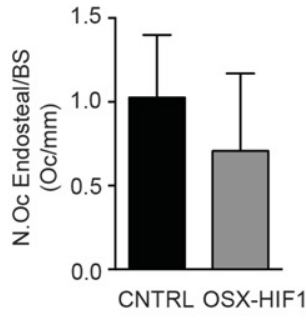

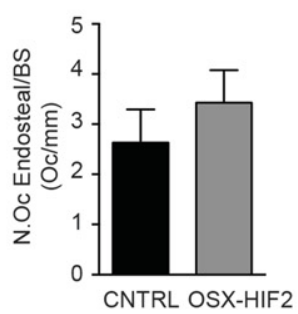

Figure 5. Loss of both HIF-1 $\alpha$ and HIF-2 $\alpha$ is necessary to alter osteoclastogenesis. $(A, B)$ Real-time PCR analysis of PGK (left) and OPG (right) mRNA expression in primary osteoblast cultures isolated from $H i f-1 \alpha^{f l / f 1}$ (HIF-1fl/fl) or HIF-2a $\alpha^{\text {fl/fl }}$ (HIF-2fl/fl) mice exposed to hypoxia $(0.5 \%$ oxygen) and infected with adenovirus to drive expression of Cre-recombinase. $(C, D)$ Real-time PCR analysis of OPG mRNA from whole-bone homogenates $(C)$ and quantification of TRAP-positive multinucleated trabecular and endosteal osteoclasts $(D)$ in proximal tibias of 12-wk-old OSX-Cre; Hif- $1 \alpha^{f l / f 1}(n=3)$. mice $(E, F)$ Real-time PCR analysis of OPG mRNA from whole-bone homogenates $(E)$ and quantification of TRAP-positive multinucleated trabecular and endosteal osteoclasts $(F)$ in proximal tibias of 12-wk-old OSX-Cre; Hif$2 a^{f l / f l}$ mice $(n=3)$. $(G)$ Representative images of TRAP-stained proximal tibias. TRAP-positive cells are stained in purple. $(H)$ Real-time PCR analysis of OPG mRNA in bone tissues isolated from 12-wk-old mice $(n=3)$. (I) Quantification of TRAP-positive trabecular and endosteal multinucleated osteoclasts in proximal tibias of 12 -wk-old mice $(n=3)$. All data are represented as mean \pm standard error of the mean (SEM). $\left.{ }^{* *}\right) P<0.01 ;\left(^{*}\right) P<0.05$, determined by Student's $t$-test. 
Next, to determine whether HIF-mediated modulation of osteoblastic OPG functionally contributes to osteoclastogenesis, HIF- $1 \alpha^{\mathrm{fl} / \mathrm{fl}}$ and HIF- $2 \alpha^{\mathrm{fl} / \mathrm{fl}}$ mice were crossed to mice expressing the Cre-recombinase under the control of the osterix promoter. Consistent with previously published results, we noted diminished trabecular bone volume in our OSX-Cre; Hif-1 $\alpha^{f l / f l}$ and OSX-Cre; Hif-1/Hif$2 a^{f l / f 1}$ mutant mice when compared with littermate controls (Supplemental Fig. S5A-C; Wang et al. 2007; Shomento et al. 2010; Rankin et al. 2012). Analysis of femurs isolated from 12-wk-old OSX-Cre; Hif- $1 \alpha^{f 1 / f l}$ mice revealed no differences in OPG mRNA expression, trabecular or endosteal osteoclast numbers, or trabecular osteoblast numbers (Fig. 5C,D; Supplemental Fig. S5D). Intriguingly, analysis of bones isolated from 12-wk-old OSX-Cre; Hif$2 a^{f l / f 1}$ mice showed that despite lowered levels of OPG mRNA, only a modest increase in trabecular and endosteal osteoclast numbers was observed; however, these differences did not reach statistical significance (Fig. 5E,F). This suggests that HIF-2 $\alpha$ is able to regulate OPG expression in vivo but that loss of HIF-2 $\alpha$ alone does not significantly impact osteoclastogenesis.

Combined loss of osteoblastic HIF-1a and HIF-2a results in diminished trabecular bone volume; however, the angiogenesis-independent roles mediating this phenotype remain unclear (Shomento et al. 2010; Rankin et al. 2012). While we did not detect differences in osteoclast numbers in either OSX-Cre; Hif- $1 \alpha^{f l / f 1}$ or OSX-Cre; Hif$2 a^{f l / f l}$ mice, we did observe an increased trend in osteoclast numbers. As such, we sought to determine whether osteoblastic inactivation of both HIF-1 $\alpha$ and HIF-2 $\alpha$ could enhance osteoclastogenesis. Examination and quantification of TRAP-stained femurs isolated from OSX-Cre; Hif- $1 \alpha^{f l / f 1}$; Hif- $2 \alpha^{f l / f 1}$ mice revealed a significant increase in trabecular osteoclast numbers and an increased trend in endosteal osteoclast numbers when compared with age- and sex-matched littermate controls (Fig. 5G,I). Real-time PCR analysis of whole-bone homogenates revealed significant differences in OPG mRNA expression between control and mutant mice (Fig. 5H). Thus, combined loss of HIF-1a and HIF-2 $\alpha$ is required to enhance osteoclastogenesis, which may be mediated in part through an OPG-dependent pathway.

\section{Osteoblastic inactivation of PHD2 and PHD3 modulates OPG to prevent bone loss}

We identified osteoblastic PHDs as regulators of both bone and blood homeostasis. Importantly, we also showed that selective inactivation of specific PHD isoforms could increase trabecular bone while avoiding the aberrant angiogenic and hematopoietic effects associated with loss of pVHL or all three PHD isoforms. Taken together, these observations raised the intriguing possibility that precise modulation of the HIF signaling pathway through the selective inhibition of osteoblastic PHDs had the potential to protect against chronic bone disorders such as osteoporosis. This could be achieved by regulating the communication between osteoblasts and osteoclasts without the detrimental effects to bone and blood tissues associated with uncontrolled HIF activation. To evaluate the potential therapeutic application of PHD2 and PHD3 inactivation for protection against pathological bone loss, we sham-operated or ovariectomized 3-mo-old control and OSX-Cre; $P h d 2^{f l / f 1}$; Phd $3^{f 1 / f 1}$ mice to determine whether PHD2 and PHD3 modulation of OPG could protect mice against bone loss. Upon ovariectomy, uterine weight was significantly diminished in both sets of ovariectomized mice when compared with sham-operated controls (Supplemental Fig. S6). Six weeks after surgery, histological and quantitative $\mu \mathrm{CT}$ analysis of control mice revealed a $50 \%$ decrease in trabecular bone volume in ovariectomized mice when compared with sham-operated controls (Fig. 6A-C). In contrast, ovariectomized OSX-Cre; Phd2 ${ }^{\text {fl }}$ ${ }^{f 1}$; $P h d 3^{f l / f 1}$ mutant mice were protected against this bone loss (Fig. 6A-C). Consistent with our previous findings, loss of PHD2 and PHD3 led to the normalization of the OPG serum levels and a re-establishment of physiologically normal numbers of osteoclasts in ovariectomized mice (Fig. 6D-F). These results demonstrate that osteoblastic inactivation of PHD2 and PHD3 has the potential to protect mice against post-ovariectomy bone loss by inhibiting the excessive and unbalanced rate of bone turnover and indicate the potential of selective PHD inhibitors as a new therapeutic modality in the treatment of osteoporosis.

\section{Discussion}

The osteoblastic niche houses cells of the osteoblastic and hematopoietic lineages residing in areas of variable oxygen tension. In this dynamic microenvironment, HIFmediated signaling is active and functionally important; however, the mechanisms by which the oxygen-sensing PHD isoenzymes regulate osteoblast function and bone homeostasis remain largely unknown. Through the use of conditional inactivating mutations, our study systematically elucidated the role of the cellular oxygen sensors in the bone, providing deep mechanistic insights into how PHD/HIF signaling regulates bone homeostasis and how this pathway can be potentially exploited for the treatment of chronic metabolic bone disorders. Here, we show a previously unreported mechanism by which PHD oxygensensing enzymes modulate trabecular bone homeostasis by controlling the dosage of HIF signaling by directing target gene expression to coordinate two important aspects of bone physiology: cross-talk between osteoblasts and osteoclasts and angiogenic/osteogenic coupling.

A recent study by Kusumbe et al. (2014) identified regionally defined areas of hypoxia within the bone marrow microenvironment and revealed that a specific subtype of endothelial cells is associated with functionally active HIF signaling to regulate the coupling of angiogenesis to osteogenesis. However, within a reduced oxygen environment, the cell-autonomous mechanism by which HIF signaling regulates osteoblast function and bone homeostasis remains poorly understood. In support of previous findings, our study shows that extreme HIF activation through genetic inactivation of all three PHDs enhances bone formation through VEGF-mediated angiogenesis. 
A

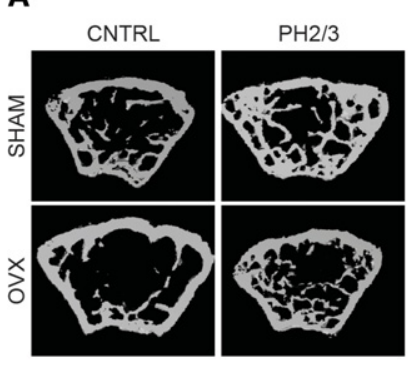

D

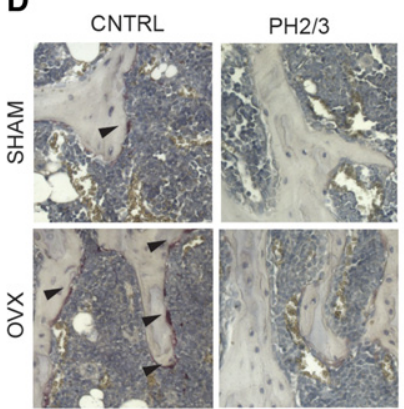

B

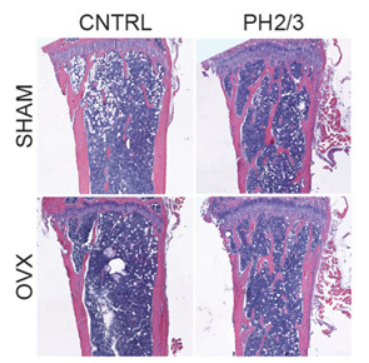

$\mathbf{E}$

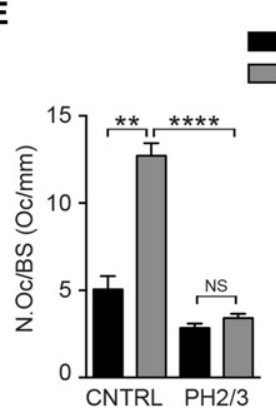

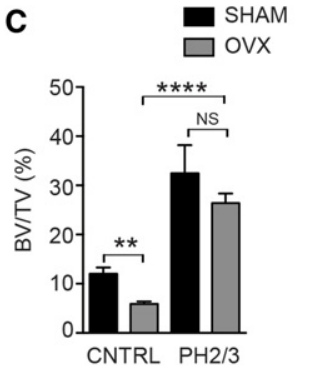

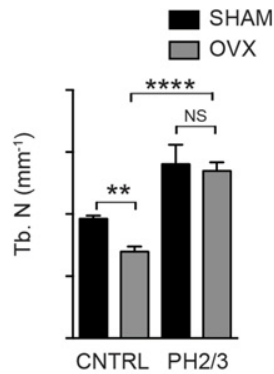

$\mathbf{F}$

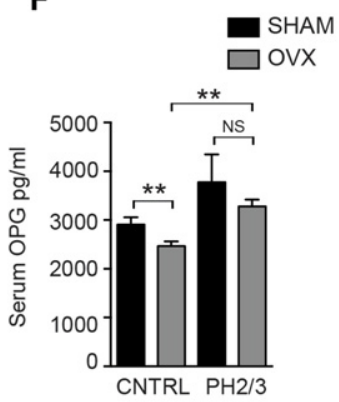

Figure 6. Osteoblastic inactivation of PHD2 and PHD3 modulates OPG to protect mice against bone loss. $(A)$ Representative $\mu C T$ images of distal femurs (axial view of metaphyseal region) isolated from sham-operated (SHAM) and ovariectomized (OVX) control (CNTRL) and OSX-Cre; $P h d 2^{f 1 / f 1}$; $P h d 3^{f l / f 1}(\mathrm{PH} 2 / 3)$ mice. (B) Representative images of H\&E-stained proximal tibias isolated from sham-operated (SHAM) and ovariectomized (OVX) control (CNTRL) and OSX-Cre; Phd2 ${ }^{f 1 / f 1}$; Phd ${ }^{f l / f 1}(\mathrm{PH} 2 / 3)$ mice. $(C) \mu \mathrm{CT}$ analysis of trabecular bone volume (BV/TV) and trabecular number (Tb.N) for sham-operated (SHAM) and ovariectomized (OVX) control (CNTRL) and OSX-Cre; Phd2 ${ }^{f l / f 1}$; $P h d 3^{f l / f 1}(\mathrm{PH} 2 / 3)$ mice. For each group analyzed, $n=9$. $(D)$ Representative images of TRAP-stained proximal tibias. The arrowheads point to TRAP-positive osteoclasts. (E) Quantification of TRAP-positive multinucleated osteoclasts in proximal tibias of sham-operated (SHAM) and ovariectomized (OVX) control (CNTRL) and OSX-Cre; Phd ${ }^{f l / f 1}$; Phd $3^{f l / f 1}$ (PHD2/3) mice. (N.Oc./BS) Number of osteoclasts per bone surface. $(F)$ Analysis of OPG protein levels in the serum of sham-operated (SHAM) or ovariectomized (OVX) control (CNTRL) and mutant mice as determined by ELISA $(n=6)$. For all experiments, operations were performed at 12 wk of age, and 6 wk post-surgery bone parameters were assessed. Data are represented as mean \pm standard error of the mean $\left.\left.(\mathrm{SEM}) .{ }^{* *}\right) P<0.01 ;{ }^{* * * *}\right) P<0.0001$, as determined by Student's $t$-test.

Here, we present evidence indicating that PHD/HIF signaling has additional functions in skeletalgenesis. Unexpectedly, the combined genetic inactivation of $P h d 2$ and Phd3 led to modest HIF target gene activation and a moderate increase in trabecular bone volume. Importantly, this phenotype was not associated with increased angiogenesis but rather the suppression of osteoblast-mediated osteoclastogenesis through the modulation of OPG. Notably, previous studies augmenting HIF signaling in bone have used genetic inactivation of pVHL or constitutive activation of HIF-1 $\alpha$, which leads to severe HIF activation (Wang et al. 2007; Regan et al. 2014). In contrast, as PHD enzymatic activity is regulated by oxygen availability, these enzymes provide the link between oxygen availability and HIF activation, and, as such, our study provides a complete and nuanced understanding of how hypoxia contributes to bone homeostasis.

Our study identified OPG as a new osteoblastic HIF target gene. We discovered functional HIF-binding sites within the Tnfrsf11b (OPG) promoter and report that constitutive activation of PHD/HIF signaling resulted in direct binding of HIF-2 $\alpha$ to these regions. We report that HIF-mediated signaling is both sufficient and necessary, in the context of PHD2 and PHD3 inactivation, to modulate OPG expression to direct osteoblast-mediated osteo- clastogenesis. We show that a twofold induction in OPG mRNA may have the potential to alter bone homeostasis. In support of our work, Movérare-Skrtic et al. (2014) also demonstrated that a twofold reduction in OPG was associated with diminished bone strength. In vivo, we found that enhanced trabecular bone volume was associated with a threefold increase in OPG mRNA expression. These changes are in accordance with other mouse models in which stabilization of $\beta$-catenin increased bone formation through the up-regulation of OPG /Glass et al. 2005). Importantly, we cannot dismiss the possibility that augmented PHD/HIF signaling may mediate other signaling events that contribute to the modulation of bone homeostasis seen in vivo. For example, the Wnt/canonical $\beta$-catenin signaling pathway has been shown to directly bind to and up-regulate OPG expression to impair bone resorption (Glass et al. 2005). While our data demonstrate a direct regulation of OPG by PHD/HIF signaling, we cannot rule out the possibility that hypoxia and HIF may also indirectly regulate OPG, osteoclastogenesis, and bone homeostasis through indirect effects on other pathways such as the $\mathrm{WNT} / \beta$-catenin pathway.

While previous reports show that osteoblastic loss of HIF- $1 \alpha$ and HIF- $2 \alpha$ results in diminished trabecular bone volume, the angiogenesis-independent roles mediating 
this phenotype remain unclear (Shomento et al. 2010). We did not detect differences in osteoclast numbers in either OSX-Cre; Hif- $1 \alpha^{f l / f 1}$ or OSX-Cre; Hif- $2 \alpha^{f l / f 1}$ mice despite a reduction in OPG mRNA in OSX-Cre; Hif- $2 \alpha^{f l / f 1}$ mice. However, combined loss of osteoblastic HIF-1 $\alpha$ and HIF$2 a$ resulted in diminished osteoclast numbers accompanied by a reduction of trabecular bone exceeding that of loss of HIF-1a alone. Importantly, Shomento et al. (2010) reported that loss of HIF-2 $\alpha$ in osteoblasts was not associated with a significant loss in trabecular bone despite a significant decrease in bone vasculature. Given these findings, we hypothesize that HIF-1 $\alpha$ under normal physiological settings may play a predominant role in regulating osteogenesis coupled to angiogenesis, and this may impact the subsequent recruitment of osteoclast precursors to areas of bone remodeling. In contrast, HIF- $2 a$ may also contribute to bone remodeling in part by regulating the interactions between osteoblasts and osteoclasts through the modulation of OPG.

Importantly, we show that PHDs in osteoblasts can modulate the cross-talk between osteoblasts and osteoclasts. This previously unreported relationship between HIF transcription factors and the RANK/RANKL/OPG signaling axis also has important clinical implications, as deregulation of the OPG/RANK/RANKL pathway is a critical molecular driver in the development of post-menopausal osteoporosis. Moreover, we demonstrated that genetic inactivation of all three PHD isoforms resulted in excessively ossified bone associated with enhanced microvascular density, reduction in bone marrow cellularity, and the development of polycythemia. Thus, for clinical purposes, long-term activation of PHD/HIF signaling within the bone marrow microenvironment has potential detrimental effects for both bone and hematopoietic tissue and should therefore be approached with caution, as unmitigated HIF activation can lead to extreme VEGF production, which has been shown to result in excessively ossified bone (Maes et al. 2010b).

However, it should be noted that transient activation of the HIF signaling pathway through pharmacologic pan-inhibition of PHDs through the use of either iron cheaters (such as desferrioxamine [DFO]) or 2-oxyglutarate analogs (such as dimethyloxalyglycine [DMOG]) increases VEGFmediated vascularization at the fracture site and promotes callus formation and efficient healing during fracture repair (Wan et al. 2008; Shen et al. 2009). Hence, for acute bone injuries, limited and transient activation of PHD/ HIF signaling may prove to be beneficial by enhancing blood vessel invasion and the recruitment of new osteoprogenitors, both of which are critical mediators for efficient and proper bone healing after fractures. Given these observations, it has been postulated that pan-PHD inhibitors could also be used for the treatment of chronic metabolic bone disorders such as osteoporosis (Zhao et al. 2012). In support of this hypothesis, DMOG has been shown to increase trabecular bone and bone strength mediated by VEGF-induced angiogenesis; however, it should be noted that these experiments were performed on rats over a relatively short period of time (Liu et al. 2013). Furthermore, in these studies, examination of the hematopoietic compartment was not performed to determine whether hematopoietic hemostasis was maintained during the course of treatment. Taken together, the efficacy of using pan-PHD inhibitors for the treatment of osteoporosis when long-term, excessive chronic exposure would be required has yet to be proven. Our findings suggest that selective rather than collective inhibition of PHD isoenzymes should be explored as protection against metabolic bone disorders to avoid the potential tissue-damaging effects of prolonged, chronic, and excessive HIF activation. In addition, selective inactivation of PHD2 and PHD3 enhances trabecular bone volume through modulation of the OPG, thereby specifically targeting the molecular mechanism driving the pathogenesis of this disease.

We show that "fine-tuning" of osteoblastic HIF signaling in bone tissue through specific PHD isoform inactivation can control the dosage of HIF signaling to differentially activate HIF target genes (Fig. 7). Importantly, precise modulation of HIF activation is associated with functional bone accumulation and hematopoietic

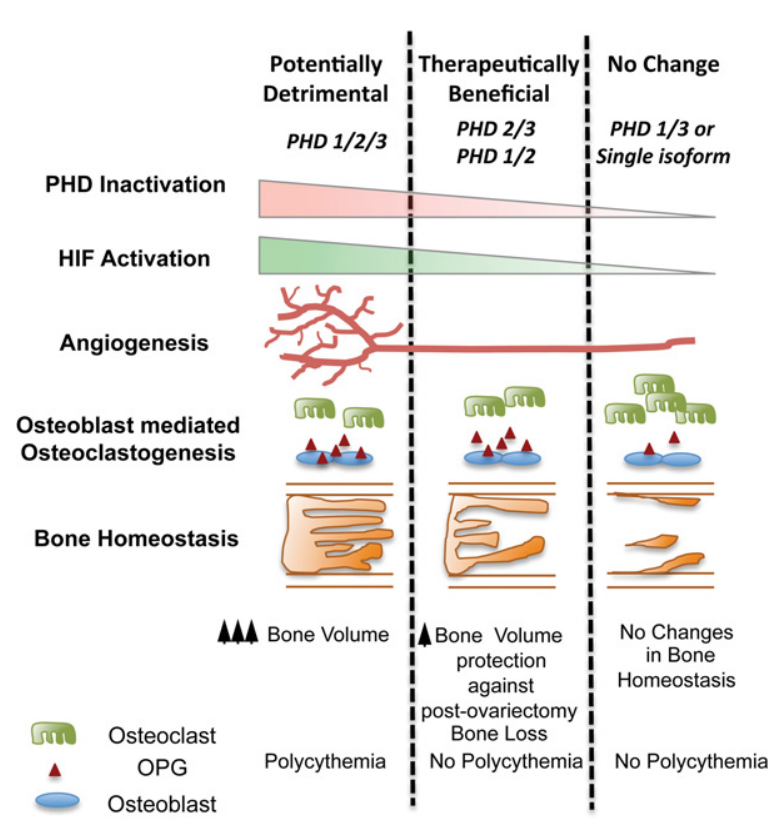

Figure 7. Selective inactivation of PHDs regulates HIF signaling dosage to differentially modulate bone homeostasis. Selective inactivation of PHDs modulates the dosage of HIF signaling with functional consequences for bone and hematopoietic homeostasis. We show that, by acting as a molecular rheostat of HIF activation, genetic inhibition of specific PHD isoform combinations differentially regulates two important aspects of bone physiology: cross-talk between osteoblasts and osteoclasts and angiogenic/osteogenic coupling. In conditions where genetic inactivation of three PHD isoforms occurs, robust HIF activation ensues, leading to enhanced angiogenesis that results in extreme ossification of trabecular bones and aberrant hematopoiesis. In contrast, the loss of specific dual combinations of PHD isoforms does not alter microvascular density or hematopoietic homeostasis but does enhance trabecular bone volume. This enhancement is due to HIFmediated suppression of osteoblast-mediated osteoclastogenesis through the modulation of OPG. 
homeostasis rather than the accumulation of trabecular bone that obliterates the bone marrow cavity. Furthermore, we were able to delineate between $\mathrm{PHD} / \mathrm{HIF}$ regulation of bone and hematopoietic homeostasis. Importantly, PHDs are current therapeutic targets for the regulation of HIF in the treatment of anemia and wound healing (Myllyharju 2008; Minamishima and Kaelin 2010). However, currently available HIF hydroxylase inhibitors lack specificity for individual PHD isoenzymes, highlighting the importance of developing isoform-specific inhibitors as a possible treatment modality to protect against osteoporosis that is not associated with the potential development of tissue toxicity (Fraisl et al. 2009). We did not detect changes in cortical bone, a large determinant of bone strength and nonvertebral fracture risk. However, trabecular bone is important for the prevention of osteoporosis, particularly for the impediment of vertebral fractures, which result in pain, functional disability, and decreased quality of life (Gerdhem 2013; Iki et al. 2014). Therefore, the resulting increases in trabecular bone volume afforded by PHD2 and PHD3 inactivation may have important clinical implications. In summary, we have begun to unravel the means by which oxygen availability and PHD oxygen sensors act as the molecular rheostat of HIF activation to differentially activate HIF target genes and functionally contribute to several physiological processes that control trabecular bone homeostasis.

\section{Materials and methods}

Generation of mice

Generation and genotyping analysis of the $P h d 1^{f 1 / f 1}$; $P h d 2^{f 1 / f 1}$; Phd $3^{f l / f 1}$, Hif-1dPA, Hif-2dPA, Hif-1 ${ }^{f l / f 1}, H i f-2^{f 1 / f 1}$, and OSX-Cre transgenic mice have previously been described (Ryan et al. 1998; Kim et al. 2006; Rodda and McMahon 2006; Takeda et al. 2006, 2008; Gruber et al. 2007). OSX-Cre; $P h d^{f l / f 1}$ mice were generated in mixed C57/B6 and FVB/N genetic backgrounds. Therefore, for all studies with these mice, littermate Cre-recombinasepositive heterozygous floxed mice and Cre-negative homozygous floxed mice were used as controls. OSX-Cre; Hif-1dPA and OSXCre; Hif-2dPA mice were bred on a C57/B6 genetic background. For all studies, age- and sex-matched littermates were used as controls. All procedures involving mice were performed in accordance with the National Institutes of Health (NIH) guidelines for the use and care of live animals and were approved by the Stanford University Institutional Animal Care and Use Committee.

\section{Cell culture}

MC3T3 E1 cells (American Type Culture Collection) were maintained in aMEM (Life Technologies) with 10\% FBS (HyClone) (v/ v) at $37^{\circ} \mathrm{C}$ in a humidified incubator supplied with $5 \% \mathrm{CO}_{2}$. Transfections were carried out using polyethylenimine (Sigma). Briefly, MC3T3 E1 cells were incubated with a ratio of $50 \mu \mathrm{g}$ of DNA: $2 \mu \mathrm{g}$ of polyethylenimine for $6 \mathrm{~h}$, after which cells were rinsed with $\alpha M E M$ and incubated for $24 \mathrm{~h}$ prior for endpoint analysis (see below). For hypoxia treatments, cells were plated $12 \mathrm{~h}$ prior to placement into a hypoxia chamber (Invivo2-400, Ruskin Technologies) and maintained at $0.5 \%$ oxygen for $24 \mathrm{~h}$. All endpoints measured for cells under hypoxic conditions were compared with cells cultured under normoxic conditions $(21 \%$ oxygen). Cocultures were prepared as previously described (Movérare-Skrtic et al. 2014). Briefly, mouse calvarias osteoblast cells from $\mathrm{PHD} 2^{\mathrm{fl} / \mathrm{fl}} ; \mathrm{PHD}^{\mathrm{fl} / \mathrm{fl}}$ mice were treated with adeno-CRE or control virus for $24 \mathrm{~h}$ as previously described (Wang et al. 2007; see below). Next, cells were treated with complete osteogenic aMEM. After $6 \mathrm{~d}$, bone marrow stromal cells from wild-type mice were added, and osteoclastogenesis was induced by culturing cells in complete osteogenic aMEM medium with $10 \mathrm{nM}$ 1,25-dihydroxy-vitamin-D3 and $1 \mu \mathrm{M} \mathrm{PGE}_{2}$.

\section{Osteoblast isolation}

Bone marrow stromal cells from postnatal day 1 pups were isolated by serial collagenase digestion as previously described $(\mathrm{Wu}$ et al. 2008). Fractions 3-6 were plated in aMEM supplemented with $10 \%$ FBS and incubated under $5 \%$ oxygen for $4 \mathrm{~d}$ to reach confluence.

\section{cDNA constructs}

pCDNA3.1 HA-Hif2 proline mutants were purchased from Addgene.

\section{Viral infections}

Primary osteoblasts from mutant mice were isolated from postnatal day 1 pups. Cells were grown to confluence and infected with control (Ad-GFP) or Cre-recombinase virus (Ad-Cre) (Vector Laboratories) at a multiplicity of infection (MOI) of 200. Total mRNA was extracted from infected osteoblasts for endpoint analysis.

\section{Protein isolation and Western blot analysis}

Protein lysates were isolated in RIPA buffer, and quantification was assessed using a Bradford assay (Bio-Rad). Fifty micrograms of protein was subjected to reducing SDS-PAGE using standard methods. Western blots were probed with antibodies against $\beta$-actin (Sigma), anti-HIF-2 (Novus), anti-HA (Sigma), anti-PHD1 (Abcam), anti-PHD2 (Novus), and anti-PHD3 (Novus). Antibodies were diluted in Superblock TBS solution (Pierce) and maintained at $4^{\circ} \mathrm{C}$.

\section{Promoter analysis and ChIP assay}

The Tnfrsf11B (OPG) promoter was searched for consensus HREs containing a conserved RCGTG sequence. Potential HREs located within the Tnfrsf $11 b$ gene and promoter regions were predicted using the JASPAR CORE database with a relative score threshold of 0.85 . ChIP assay was performed as described previously with the following modifications (Krieg et al. 2010). MC3T3-E1 cells were fixed $24 \mathrm{~h}$ after transfection with control or pcDNA 3.1 HIF-2-HA constructs (Addgene). Lysed cells were sonicated using a Bioruptor plus sonication system (Diagenode, Inc.) set at high power, $30 \mathrm{sec}$ on, $90 \mathrm{sec}$ off, and 60 cycles. Approximately $150 \mu \mathrm{g}$ of sonicated chromatin was incubated overnight with rabbit anti-HA antibody (Abcam) followed by precipitation with protein A/G Dynabeads (Invitrogen). Normal rabbit IgG (Santa Cruz Biotechnology) was used as a nonspecific IgG control. Five percent of the sample from each immunoprecipitation was reserved for input control. Relative enrichment was measured by qRT-PCR using a titration of pooled input samples as a standard curve and normalized to input after subtraction of IgG signal. Enrichments are presented as fold change relative to mock control. 
Wu et al.

\section{Quantitative $\mu$ CT analysis}

Femurs and tibias from 12-wk-old mice were dissected, soft tissue was removed, and bones were fixed in 10\% buffered formalin for 5 $\mathrm{d}$ and then stored in $70 \%$ ethanol. Ex vivo specimens were scanned at a voxel size of $10.5 \mu \mathrm{m}$ ( $\mu$ CT40, Scanco Medical). Trabecular bone analysis of distal femurs was performed using acquired images beginning $0.5 \mathrm{~mm}$ below the distal growth plate and extending proximally for $2.0 \mathrm{~mm}$. For trabecular bone analysis of tibias, analysis was performed over a $1.3-\mathrm{mm}$ region beginning $0.4 \mathrm{~mm}$ below the proximal growth plate. Cortical bone analysis was performed using $1.0-\mathrm{mm}$ transverse cross-sectional sections in the mid-diaphyseal region of the femur. For trabecular bones, each $\mu \mathrm{CT}$ image shown represents a $10-\mu \mathrm{m}$ transverse slice $0.8 \mathrm{~mm}$ below the femoral distal growth plate. We applied a threshold of 170 to all scans at high resolution $(E=55 \mathrm{kVp}, I=$ $72 \mu \mathrm{A}$, integration time $=200 \mathrm{msec}$ ), and standard algorithms were used to calculate the following parameters: bone volume/total volume (BV/TV), trabecular number (Tb.N.), trabecular thickness (Tb.Th.), trabecular separation (Tb.Sp.), cortical thickness (Ct.Th.), total cross-sectional area (Tt.Ar), cortical bone area (Ct.Ar), medullary area, and cortical area fraction (Ct.Ar/Tt.Ar).

\section{Histomorphometry and immunohistochemistry}

For dynamic histomorphometry, calcein green was injected on day 1 followed by a second injection on day 7. Mice were euthanized $2 \mathrm{~d}$ after the second injection. Undecalcifed femurs were embedded in methymethacrylate. In vivo osteoclast and osteoblast parameters were generated from paraffin sections of decalcified tibias stained with TRAP or H\&E, and images were analyzed using Bioquant software (Bioquant Image Analysis). Osteoclast number normalized to the bone surface (N.Oc/Bs) was quantified on TRAP-stained sections according to the manufacturer's instructions (Sigma) at 200× magnification. All measurements were taken in an area located $0.5 \mathrm{~mm}$ below the growth plates. HIF- $1 \alpha$ and HIF- $2 \alpha$ were detected with rabbit anti-human/mouse HIF-2 $\alpha$ polyclonal antibody (Novus) and rabbit anti-human/ mouse HIF-1 $a$ (Bethyl Laboratories) at a dilution of 1:100. CD31 was detected with rat anti-mouse CD31 antibody (BD 550274) at a dilution of 1:200. Endomucin was detected with rat antimouse endomucin antibody (Abcam) at a dilution of 1:50. All antibodies were incubated overnight at $4^{\circ} \mathrm{C}$ and developed with the TSA biotin system (Perkin-Elmer) according to the manufacturer's protocol.

\section{Ovariectomy-induced bone loss}

Twelve-week-old female mice were ovariectomized or sham-operated. Six weeks after surgery, mice were euthanized and subjected to bone analysis as described above.

\section{DNA and RNA isolation}

DNA was isolated according to Laird et al. (1991) and used for genomic PCR. RNA was isolated using Trizol reagent according to the manufacturer's instructions (Invitrogen).

\section{Real-time PCR}

Real-time PCR was performed as previously described (Rankin et al. 2012). For $18 \mathrm{~S}$ amplification, cDNA was diluted 1:50. The following primers were used to amplify specific target genes: Epo (FWD, 5'-CATCTGCGACAGTCGAGTTCTG-3' and REV, 5'-CACAACCCATCGTGACATTTT C-3' ), 18 S (FWD, 5-GCCC
GAAGCGTTTACTTTGA-3' and REV, 5-TCCATTATTCCTA GCTGCGGTATC- $3^{\prime}$ ), Pgk (FWD, 5'-GGAAGCGGGTCGTGA TGA-3' and REV, 5'-GCCTTGATCCTTTGGTTGTTTG-3'), Vegf (FWD, 5'-CCACGTCAGAGAGCAACATCA-3' and REV, 5'-TCATTCTCTCTATGTGCTGGCTTT-3'), Opg (FWD, 5'-A GCTGCTGAAGCTGTGGAA-3' and REV, 5'-GGTTCGAGT GGCCGAGAT-3'), and Rankl (FWD, 5'-ATGAACAAGTGGCT GTGCTG-3' and REV, 5' ${ }^{\prime}$-TGTCACACAGGAGCTGATGA-3').

\section{ELISA}

OPG and RANKL ELISA was performed according to the manufacturer's instructions with the exception that the serum was incubated in the ELISA plate overnight at $4^{\circ} \mathrm{C}$ (R\&D Systems).

\section{Statistical analysis}

Statistical analysis was performed using Prism software (GraphPad). In all cases, a two-tailed, unpaired Student's $t$-test was performed to analyze the statistical differences between groups. $P$-values of $<0.05$ were considered statistically significant.

\section{Acknowledgments}

We thank all of the investigators who generated and made the floxed/transgenic mice available for these studies: Dr. Brian Keith and Dr. Celeste Simon (Hif-2 and Hif-1 floxed), Dr. Guo-Hua Fong (Phd1 floxed, Phd2 floxed, and Phd3 floxed), and Dr. Andrew McMahon (OSX-Cre). This work was supported by National Institutes of Health grants CA088840, CA67166, and AR048191 and the Skippy Frank Translational Medicine and Life Sciences Fund of Rockefeller Philanthropy Advisors. C.W. is supported by a Canadian Institute of Health Research Fellowship.

\section{References}

Boyle WJ, Simonet WS, Lacey DL. 2003. Osteoclast differentiation and activation. Nature 423: 337-342.

Chow DC, Wenning LA, Miller WM, Papoutsakis ET. 2001. Modeling $\mathrm{pO}(2)$ distributions in the bone marrow hematopoietic compartment. I. Krogh's model. Biophys J 81: 675-684.

Fraisl P, Aragones J, Carmeliet P. 2009. Inhibition of oxygen sensors as a therapeutic strategy for ischaemic and inflammatory disease. Nat Rev Drug Discov 8: 139-152.

Gerdhem P. 2013. Osteoporosis and fragility fractures: vertebral fractures. Best Pract Res Clin Rheumatol 6: 743-755.

Glass DA II, Bialek P, Ahn JD, Starbuck M, Patel MS, Clevers H, Taketo MM, Long F, McMahon AP, Lang RA, et al. 2005. Canonical Wnt signaling in differentiated osteoblasts controls osteoclast differentiation. Dev Cell 8: 751-764.

Gruber M, Hu CJ, Johnson RS, Brown EJ, Keith B, Simon MC. 2007. Acute postnatal ablation of Hif- $2 \alpha$ results in anemia. Proc Natl Acad Sci 104: 2301-2306.

Iki M, Tamaki J, Kadowaki E, Sato Y, Dongmei N, Winzenrieth R, Kagamimori S, Kagawa Y, Yoneshima H. 2014. Trabecular bone score (TBS) predicts vertebral fractures in Japanese women over 10 years independently of bone density and prevalent vertebral deformity: the Japanese population-based osteoporosis (JPOS) cohort study. J Bone Miner Res 29: 399-407.

Khosla S, Westendorf JJ, Oursler MJ. 2008. Building bone to reverse osteoporosis and repair fractures. J Clin Invest 118: 421-428.

Kim WY, Safran M, Buckley MR, Ebert BL, Glickman J, Bosenberg M, Regan M, Kaelin WG Jr. 2006. Failure to prolyl hydroxylate 
hypoxia-inducible factor a phenocopies VHL inactivation in vivo. EMBO I 25: 4650-4662.

Kondo K, Klco J, Nakamura E, Lechpammer M, Kaelin WG Jr. 2002. Inhibition of HIF is necessary for tumor suppression by the von Hippel-Lindau protein. Cancer Cell 1: 237-246.

Krieg AJ, Rankin EB, Chan D, Razorenova O, Fernandez S, Giaccia AJ. 2010. Regulation of the histone demethylase JMJD1A by hypoxia-inducible factor 1a enhances hypoxic gene expression and tumor growth. Mol Cell Biol 30: 344-353.

Kronenberg HM. 2003. Developmental regulation of the growth plate. Nature 423: 332-336.

Kusumbe AP, Ramasamy SK, Adams RH. 2014. Coupling of angiogenesis and osteogenesis by a specific vessel subtype in bone. Nature 507: 323-328.

Laird PW, Zijderveld A, Linders K, Rudnicki MA, Jaenisch R, Berns A. 1991. Simplified mammalian DNA isolation procedure. Nucleic Acids Res 19: 4293.

Liu X, Tu Y, Zhang L, Qi J, Ma T, Deng L. 2013. Prolyl hydroxylase inhibitors protect from the bone loss in ovariectomy rats by increasing bone vascularity. Cell Biochem Biophys 69: 141-149.

Maes C. 2013. Role and regulation of vascularization processes in endochondral bones. Calcif Tissue Int 92: 307-323.

Maes C, Kobayashi T, Selig MK, Torrekens S, Roth SI, Mackem S, Carmeliet G, Kronenberg HM. 2010a. Osteoblast precursors, but not mature osteoblasts, move into developing and fractured bones along with invading blood vessels. Dev Cell 19:329-344.

Maes C, Goossens S, Bartunkova S, Drogat B, Coenegrachts L, Stockmans I, Moermans K, Nyabi O, Haigh K, Naessens M, et al. 2010b. Increased skeletal VEGF enhances $\beta$-catenin activity and results in excessively ossified bones. EMBO J 29: 424-441.

Minamishima YA, Kaelin WG Jr. 2010. Reactivation of hepatic EPO synthesis in mice after PHD loss. Science 329: 407.

Mohyeldin A, Garzon-Muvdi T, Quinones-Hinojosa A. 2010. Oxygen in stem cell biology: a critical component of the stem cell niche. Cell Stem Cell 7: 150-161.

Movérare-Skrtic S, Henning P, Liu X, Nagano K, Saito H, Börjesson AE, Sjögren K, Windahl SH, Farman H, Kindlund B, et al. 2014. Osteoblast-derived WNT16 represses osteoclastogenesis and prevents cortical bone fragility fractures. Nat Med 11: 1279-1288.

Myllyharju J. 2008. Prolyl 4-hydroxylases, key enzymes in the synthesis of collagens and regulation of the response to hypoxia, and their roles as treatment targets. Ann Med 40: 402-417.

Provot S, Schipani E. 2005. Molecular mechanisms of endochondral bone development. Biochem Biophys Res Commun 328: 658-665.

Rankin EB, Giaccia AJ, Schipani E. 2011. A central role for hypoxic signaling in cartilage, bone, and hematopoiesis. Curr Osteoporos Rep 9: 46-52.

Rankin EB, Wu C, Khatri R, Wilson TL, Andersen R, Araldi E, Rankin AL, Yuan J, Kuo CJ, Schipani E, et al. 2012. The HIF signaling pathway in osteoblasts directly modulates erythropoiesis through the production of EPO. Cell 149: 63-74.

Regan JN, Lim J, Shi Y, Joeng KS, Arbeit JM, Shohet RV, Long F. 2014. Up-regulation of glycolytic metabolism is required for HIF1a-driven bone formation. Proc Natl Acad Sci 111: 8673-8678.

Rodda SI, McMahon AP. 2006. Distinct roles for Hedgehog and canonical Wnt signaling in specification, differentiation and maintenance of osteoblast progenitors. Development 133: 3231-3244.

Ryan HE, Lo J, Johnson RS. 1998. HIF-1a is required for solid tumor formation and embryonic vascularization. EMBO $J$ 17: 3005-3015.
Schipani E, Maes C, Carmeliet G, Semenza GL. 2009. Regulation of osteogenesis-angiogenesis coupling by HIFs and VEGF. I Bone Miner Res 24: 1347-1353.

Schofield CJ, Ratcliffe PJ. 2004. Oxygen sensing by HIF hydroxylases. Nat Rev Mol Cell Biol 5: 343-354.

Seeman E, Delmas PD. 2006. Bone quality-the material and structural basis of bone strength and fragility. N Engl J Med 354: 2250-2261.

Semenza GL. 2001. HIF-1, $\mathrm{O}_{2}$, and the 3 PHDs: how animal cells signal hypoxia to the nucleus. Cell 107: 1-3.

Semenza GL. 2012. Hypoxia-inducible factors in physiology and medicine. Cell 148: 399-408.

Shen X, Wan C, Ramaswamy G, Mavalli M, Wang Y, Duvall CL, Deng LF, Guldberg RE, Eberhart A, Clemens TL, et al. 2009. Prolyl hydroxylase inhibitors increase neoangiogenesis and callus formation following femur fracture in mice. J Orthop Res 27: 1298-1305.

Shomento SH, Wan C, Cao X, Faugere MC, Bouxsein ML, Clemens TL, Riddle RC. 2010. Hypoxia-inducible factors $1 a$ and $2 a$ exert both distinct and overlapping functions in long bone development. J Cell Biochem 109: 196-204.

Spencer JA, Ferraro F, Roussakis E, Klein A, Wu J, Runnels JM, Zaher W, Mortensen LJ, Alt C, Turcotte R, et al. 2014. Direct measurement of local oxygen concentration in the bone marrow of live animals. Nature 508: 269-273.

Takeda K, Ho VC, Takeda H, Duan LJ, Nagy A, Fong GH. 2006. Placental but not heart defects are associated with elevated hypoxia-inducible factor a levels in mice lacking prolyl hydroxylase domain protein 2. Mol Cell Biol 26: 8336-8346.

Takeda K, Aguila HL, Parikh NS, Li X, Lamothe K, Duan LJ, Takeda H, Lee FS, Fong GH. 2008. Regulation of adult erythropoiesis by prolyl hydroxylase domain proteins. Blood 111: 3229-3235.

Teitelbaum SL, Ross FP. 2003. Genetic regulation of osteoclast development and function. Nat Rev Genet 4: 638-649.

Vaupel P, Kallinowski F, Okunieff P. 1989. Blood flow, oxygen and nutrient supply, and metabolic microenvironment of human tumors: a review. Cancer Res 49: 6449-6465.

Wan C, Gilbert SR, Wang Y, Cao X, Shen X, Ramaswamy G, Jacobsen KA, Alaql ZS, Eberhardt AW, Gerstenfeld LC, et al. 2008. Activation of the hypoxia-inducible factor- $1 \alpha$ pathway accelerates bone regeneration. Proc Natl Acad Sci 105: 686-691.

Wang Y, Wan C, Deng L, Liu X, Cao X, Gilbert SR, Bouxsein ML, Faugere MC, Guldberg RE, Gerstenfeld LC, et al. 2007. The hypoxia-inducible factor a pathway couples angiogenesis to osteogenesis during skeletal development. J Clin Invest 117: 1616-1626.

Winkler IG, Barbier V, Wadley R, Zannettino AC, Williams S, Lévesque JP. 2010. Positioning of bone marrow hematopoietic and stromal cells relative to blood flow in vivo: serially reconstituting hematopoietic stem cells reside in distinct nonperfused niches. Blood 116: 375-385.

Wu JY, Purton LE, Rodda SJ, Chen M, Weinstein LS, McMahon AP, Scadden DT, Kronenberg HM. 2008. Osteoblastic regulation of $\mathrm{B}$ lymphopoiesis is mediated by $\mathrm{G}_{\mathrm{s}} \mathrm{\alpha}$-dependent signaling pathways. Proc Natl Acad Sci 105: 16976-16981.

Wu JY, Scadden DT, Kronenberg HM. 2009. Role of the osteoblast lineage in the bone marrow hematopoietic niches. I Bone Miner Res 24: 759-764.

Zaidi M. 2007. Skeletal remodeling in health and disease. Nat Med 13: 791-801.

Zhao Q, Shen X, Zhang W, Zhu G, Qi J, Deng L. 2012. Mice with increased angiogenesis and osteogenesis due to conditional activation of HIF pathway in osteoblasts are protected from ovariectomy induced bone loss. Bone 50: 763-770. 


\section{Corrigendum}

Genes \& Development 29: 817-831 (2015)

Corrigendum: Oxygen-sensing PHDs regulate bone homeostasis through the modulation of osteoprotegerin

Colleen Wu, Erinn B. Rankin, Laura Castellini, Javier F. Alcudia, Edward L. LaGory, Rebecca Andersen, Steven D. Rhodes, Tremika L.S. Wilson, Khalid S. Mohammad, Alesha B. Castillo, Theresa A. Guise, Ernestina Schipani, and Amato J. Giaccia

In the above-mentioned article, the authors erroneously presented the name of one author as Javier Fernandez-Alcudia. The correct name should be Javier F. Alcudia, as shown above.

The authors apologize for any confusion this may have caused.

doi: $10.1101 / \operatorname{gad} .265363 .115$ 


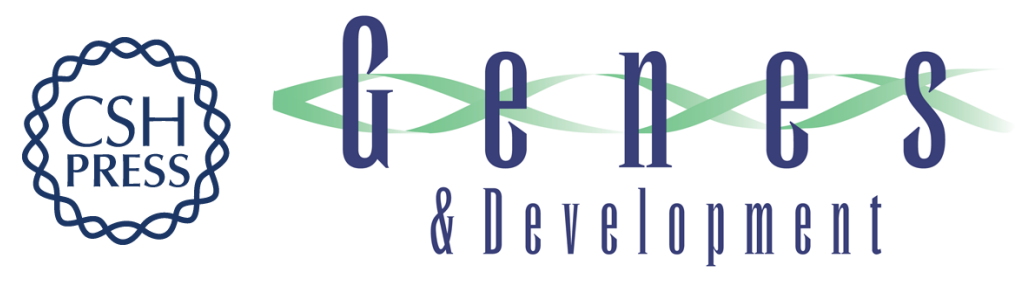

\title{
Oxygen-sensing PHDs regulate bone homeostasis through the modulation of osteoprotegerin
}

\author{
Colleen Wu, Erinn B. Rankin, Laura Castellini, et al. \\ Genes Dev. 2015, 29: originally published online April 6, 2015 \\ Access the most recent version at doi:10.1101/gad.255000.114
}

\section{Supplemental http://genesdev.cshlp.org/content/suppl/2015/04/03/gad.255000.114.DC1 \\ Material}

Related Content

Corrigendum: Oxygen-sensing PHDs regulate bone homeostasis through the modulation of osteoprotegerin

Colleen Wu, Erinn B. Rankin, Laura Castellini, et al.

Genes Dev. June , 2015 29: 1202

References This article cites 47 articles, 14 of which can be accessed free at: http://genesdev.cshlp.org/content/29/8/817.full.html\#ref-list-1

Articles cited in: http://genesdev.cshlp.org/content/29/8/817.full.html\#related-urls

Creative This article is distributed exclusively by Cold Spring Harbor Laboratory Press for the first Commons six months after the full-issue publication date (see

License http://genesdev.cshlp.org/site/misc/terms.xhtml). After six months, it is available under a Creative Commons License (Attribution-NonCommercial 4.0 International), as described at http://creativecommons.org/licenses/by-nc/4.0/.

Email Alerting Receive free email alerts when new articles cite this article - sign up in the box at the top Service right corner of the article or click here.

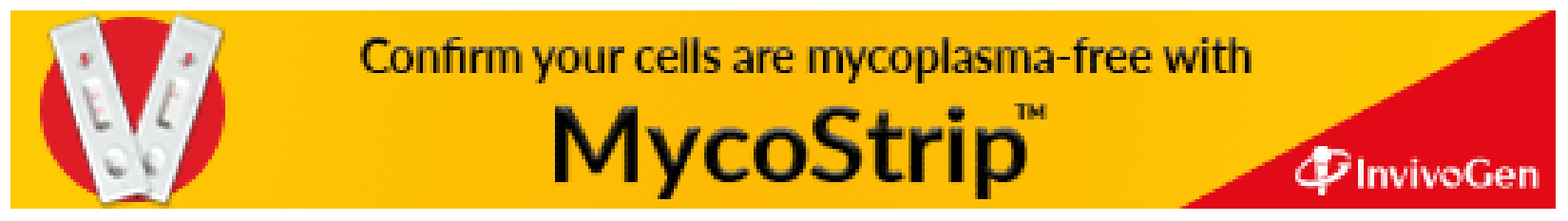

